\title{
Amplified Antitumor Efficacy By A Targeted Drugs Retention and Chemosensitization Strategies-Based "Combo" Nanoagent Together With PD-L1 Blockade In Reversing Multidrug Resistance
}

\section{Weixi Jiang ( $\square$ doctorxipishi@163.com )}

Chongqing Medical University https://orcid.org/0000-0002-7017-4570

\section{Lei Su}

Chongqing Medical University

\section{Meng Ao}

Chongqing Medical University

\section{Xun Guo}

Chongqing Medical University

\section{Chen Cheng}

Chongqing Medical University

\section{Yuanli Luo}

Chongqing Medical University

\section{Zhuoyan Xie}

Chongqing Medical University

\section{Xingyue Wang}

Chongqing Medical University

\section{Junrui Wang}

Chongqing Medical University

\section{Shuling Liu}

Chongqing Medical University

\section{Yang Cao}

Chongqing Medical University

\section{Pan Li}

Chongqing Medical University

\section{Zhigang Wang}

Chongqing Medical University

\section{Haitao Ran}

Chongqing Medical University Jianli Ren 


\section{Zhiyi Zhou}

University of Chinese Academy of Sciences

\section{Research Article}

Keywords: multidrug resistance, endo/lysosomal escape, tumor homing-penetrating peptide, chemotherapy enhancement, PD-L1 blockade

Posted Date: May 13th, 2021

DOl: https://doi.org/10.21203/rs.3.rs-492081/v1

License: (c) (i) This work is licensed under a Creative Commons Attribution 4.0 International License. Read Full License

Version of Record: A version of this preprint was published at Journal of Nanobiotechnology on July 5th, 2021. See the published version at https://doi.org/10.1186/s12951-021-00947-9. 


\section{Abstract}

Background: Recent studies have demonstrated that multidrug resistance (MDR) plays a critical role in the low efficiency of cancer chemotherapy. The main mechanism of MDR arises from the overexpression of P-glycoprotein (P-gp), which actively enhances drug efflux and limits the effectiveness of chemotherapeutic agents.

Results: In this study, we fabricated a "combo" nanoagent equipped with triple synergistic strategies for enhancing antitumor efficacy against MDR cells. Tumor homing-penetrating peptide endows the nanosystem with targeting and penetrating capabilities in the first stage of tumor internalization. The abundant amine groups of polyethylenimine (PEI)-modified nanoparticles then trigger a proton sponge effect to promote endo/lysosomal escape that enhances the intracellular accumulation and retention of anticancer drugs. Furthermore, copper tetrakis(4-carboxyphenyl)porphyrin (CuTCPP) encapsulated in the nanosystem, effectively scavenges endogenous glutathione (GSH) to relieve the detoxification mediated by GSH and sensitize the cancer cells to drugs, while simultaneously serving as a photoacoustic imaging (PAI) contrast agent for image visualization. Moreover, we also verify that the versatile nanoparticles in combination with PD-1/PD-L1 blockade therapy can not only activate immunological responses but also inhibit P-gp expression to obliterate primary and metastatic tumor.

Conclusion: This work shows a significant enhancement in therapeutic efficacy against MDR cells and syngeneic tumors compared to an equivalent dose of free paclitaxel by using multiple MDR reversing strategies.

\section{Introduction}

Although chemotherapy remains an effective approach to inhibit drug-sensitive cancer cells, a common major obstacle that emerges when using chemotherapeutic agents is intrinsic or acquired multidrug resistance $(M D R)[1,2]$. The main mechanism of MDR is due to the overexpression of P-glycoprotein ( $P$ gp) or other multidrug resistance protein (MRP)-associated efflux pumps. Under selection pressure, these efflux pumps enhance drug efflux via membrane transporters, export antitumor drugs and prevent effective cellular uptake[3, 4]. Reducing the intracellular retention and concentration of drugs in cancer cells with MDR makes chemotherapy inefficient and fails to meet the clinical demand. Although MDR inhibitors are one of most commonly used strategies to overcome MDR, nonspecific agent might downregulate P-gp expression in the cells of normal tissues that play a physiological role in excreting xenobiotics, probably leading to the destruction of homeostasis[5]. Currently, various nanosized drug carriers based on inorganic compounds, liposomes, polymers and their hybrids have been systematically investigated and drawn increasing attention because they offer plentiful favorable properties such as multifunctionalization and tumor microenvironment (TME) stimuli responsiveness[6-8]. However, these nanoparticles (NPs) with particle sizes ranging from $50 \mathrm{~nm}$ to $500 \mathrm{~nm}$ are mostly internalized via endocytosis and trapped in recycling endosomes ( $\mathrm{pH}$ 5.5-6.5) $[9,10]$. Afterwards, some of the endosomes are either transported out of cells by fusion with the plasma membrane in the form of exosomes or 
integrated with lysosomes, whose contents can be quickly deactivated in the latter case[11, 12]. Therefore, reduced accessibility of the hydrophobic chemotherapeutic agents to their target sites is found due to endo/lysosomal sequestration, resulting in marked drug resistance. Another chemotherapeutic challenge in MDR cells is a mass of cytosolic antioxidants, typically glutathione (GSH)[13-15]. As the most abundant nonprotein thiol found in all cellular compartments, GSH presents mostly in the cytoplasm (85\%) in its reduced form and is a major cellular redox component in living organisms $[16,17]$. It has been demonstrated that endogenous GSH has an upregulated concentration (up to $10 \mathrm{mM}$ ) in many drug-resistant tumor cells, which is notably higher than that in normal cells (approximately 2- to 4fold higher)[18]. By reducing or binding to chemotherapeutic agents such as paclitaxel (PTX), doxorubicin (DOX), or cisplatin, GSH-mediated detoxification plays a critical role in protecting important cellular components, preventing damage to DNA or proteins and inducing the loss of drug efficacy[19-21].

Numerous studies concentrating on the reversal of MDR have shown moderate progress[22-24], however, these notable results are restricted by a single reversal strategy that acts on only one mechanism of drug resistance. Thus, multiple synergistic strategies are urgently required to overcome various resistance mechanisms, such as the low intracellular retention of drugs and plentiful GSH in MDR cells.

Polyethylenimine (PEI) is generally recognized as an effective material that can facilitate endo/lysosomal escape due to its high amine density and buffering capacity. Compared to commoly used PEI (Mw: 25000 $\mathrm{Da})$, low molecular weight PEI (Mw: $1800 \mathrm{Da}$ ) has been suggested to possess better biocompatibility and degradability $[25,26]$. Under the weakly acidic conditions of tumor cells, the abundant amine groups in PEI can be protonated, which triggers the large inflow of protons, chloride counterions, and water into the endo/lysosomes, promoting endo/lysosomal rupture through high osmotic pressure which is known as the "proton-sponge effect"[27]. This trait is helpful to enhance the intracellular retention of drugs loaded by nanocarriers after endocytosis and avoid the enzymatic degradation of drugs by lysosomes[28]. Besides, a GSH consumption strategy was simultaneously performed in this study. An increasing amount of evidence has suggested that CuTCPP, consisting of $\mathrm{Cu}^{2+}$ and tetrakis(4-carboxyphenyl)porphyrin (TCPP) ligands, has a distinct GSH scavenging effect through the cycle conversion of $\mathrm{Cu}^{2+}$ into $\mathrm{Cu}^{1+}$ in CuTCPP[29-31]. Thus, CuTCPP was encapsulated into the core of poly(lactic acid-co-glycol acid) (PLGA)based NPs to sensitize cancer cells to chemotherapy, the so-called chemosensitization, via $\mathrm{Cu}^{2+}$-induced GSH depletion along with a pro-oxidant[32]. In addition, because of good optical absorption properties in the near infrared (NIR) region, CuTCPP is considered as an excellent candidate for photoacoustic imaging (PAI), a promising imaging mode due to its noninvasiveness and high spatial resolution. It is therefore believed that a nanoplatform containing CuTCPP can serve as a favourable PAI enhanced-contrast agent for image visualization and monitoring during the process of treatment[33]. We have previously reported a series of tumor homing-penetrating peptide (THPP)-functionalized NPs that specifically recognize the endothelium of tumor vessels and transport the attached cargo into the cells[34, 35]. As 7 amino acid truncated form peptide of LyP1(CGNKRTRGC), tLyP-1 (CGNKRTR) has been demonstrated to be a ligand that targets to the neuropilin-1 (NRP-1) receptor, which is overexpressed in breast cancer and several human tumor types. Moreover, by actively transferring through tumor tissue barriers, such as extracellular matrix barrier and vascular endothelial barrier, the emerging peptide is readily promote NPs to permeate 
deeper into solid tumor regions where lack microvessels for further enhancement of reversal efficiency[36, 37]. In this case, we expect this powerful THPP to endow the nanosystem with tumor targeting and penetrating abilities in the first stage of tumor internalization to assist in overcoming MDR.

Notably, the use of different types of cancer immunotherapies including cancer vaccines, cytokine therapy, adoptive T-cell transfer and checkpoint blockade have achieved exciting outcomes in clinics[38, 39]. A recent study revealed that PD-1/PD-L1 inhibition may down-regulate PI3K/AKT and MAPK/ERK pathways mediated MDR1/P-gp expression in breast cancer cells, implying this probably contribute to increase chemotherapy efficacy in breast cancer patients[40]. On the other hand, it has been reported that secretion of pro-inflammatory cytokines or chemokines such as tumor necrosis factor (TNF), alphainterferon (IFN) and interleukin-6 (IL-6) triggered by immune system is also related to expression of various MRP types. Although immune therapy for overcoming MDR tumors is not an optimal selection at this early juncture, immune-chemotherapy as a promising strategy combining cytotoxic agents with immune strategy for combating MDR tumors appears feasible in theory[41].

Taken together, herein, we synthesized the versatile NPs namely, tLyP-1-PEI-PLGA-PTX-CuTCPP (TPP@PTX-CuTCPP), which are equipped with triple MDR reversal strategies for tumor chemotherapy amplification as demonstrated in Scheme 1. Meanwhile, the strategy that multifunctional NPs further combined with PD-L1 checkpoint blockade, which expects to play a function of suppressing P-gp expression and activation of cancer immunotherapy, was developed. The anticancer efficacy and imaging ability of these NPs were evaluated in Vitro and in Vivo.

\section{Experimental}

\subsection{Materials and Reagents:}

poly(lactic-co-glycolic acid) (PLGA, lactide: glycolide =50:50, molecular weight [MW] 15,000 Da) was purchased from Jinan Daigang Biomaterial Co., Ltd. (Shandong, China). poly(vinyalcohol) (PVA, MW 25,000 Da) and Polyethylenimine (PEI, MW 1,800 Da) were purchased from Sigma-Aldrich (St Louis, MO, USA). Paclitaxel (PTX) was purchased from Aladdin Bio-Chem Technology Co., Ltd (Shanghai, China). Cutetrakis(4-carboxyphenyl)porphyrin and FITC-labeled PTX were purchased from Ruixi Biotech Co., Ltd (Xi'an, China). tLyP-1 (CGNKRTR) was synthesized by Chinapeptide (Shanghai, China). MCF-7, MCF7/Taxol and HUVECs were purchased from the MEIXUAN Biological science and technology Co., Ltd (Shanghai, China). GSH assay kit were purchased from Nanjing Jiancheng Bioengineering Institute (Nanjing, China). LysoTracker Green DND-26 and agarose were purchased from Invitrogen (Thermo Fisher Scientific). Cell Counting Kit-8 (CCK-8) Calcein-AM, and PI were obtained from Dojindo (Japan). P-gp Rabbit pAb were obtained from ABclonal (Wuhan, China). Anti-PD-L1 was purchased from BioXcell (USA). Tumor necrosis factor alpha, interferon gamma and interleukin 6 ELISA kit were purchased from Uscn Life Science, Inc., (China). Anti-CD3 ${ }^{+}-$FITC, anti-CD8 ${ }^{+}$a-APC and anti-CD $4^{+}-P C 5.5$ were purchased from Biolegend (USA). Deionized water obtained from the Millipore system (Direct-Q 5, FRA) was used in all preparations. 


\subsection{Synthesis of tLyP-1-PEI}

$20 \mathrm{mg}$ tLyP-1 was first dissolved completely in $5 \mathrm{~mL}$ of methanol, followed by the addition of 6 - (maleic imide) caproic acid succinimide ester $(40.69 \mathrm{mg}, 0.132 \mathrm{mmol})$ at room temperature for $30 \mathrm{~min}$. Subsequently, PEI $(237.6 \mathrm{mg}, 0.132 \mathrm{~mol})$ and triethylamine $\left(0.072 \mathrm{~mol} \mathrm{~L}^{-1}, 5 \mathrm{~mL}\right)$ were added into the reaction at room temperature for another $1 \mathrm{~h}$. Finally, organic solvents were removed by vacuum-rotary evaporation procedure and the intermediate solution was dialysed ( $\mathrm{MW}$, cut-off $=3,500 \mathrm{Da}$ ) against distilled water for $48 \mathrm{~h}$ to remove untreated stuff and lyophilization. The product was denoted as tLyP-1PEI.

\subsection{Synthesis of TPP@PTX-CuTCPP}

The nanoparticles encapsulating PTX and CuTCPP were synthesized by using a modified double emulsion strategy. Initially, PTX (5 mg) was added into PLGA $(50 \mathrm{mg})$ dissolved in trichloromethane (2 $\mathrm{mL})$ as the stock solution and CuTCPP aqueous solution $\left(400 \mu \mathrm{L}, 12.5 \mathrm{mg} \mathrm{mL}^{-1}\right)$ was then put in the above solution. Afterwards, the mixture was firstly emulsified through ultrasonic probe (Sonics \& Materials Inc., USA) with power of $75 \mathrm{~W}$ for $3 \mathrm{~min}$. For the second emulsion, PVA solution $(5.5 \mathrm{~mL}, \mathrm{~W} / \mathrm{v}=$ $4 \%$ ) was poured into above solution for homogenization at $45 \mathrm{~W}$ for $2 \mathrm{~min}$. Isopropyl alcohol solution $(12.5 \mathrm{ml}, \mathrm{v} / \mathrm{v}=2 \%)$ was then poured in final emulsion to evaporate organic solvent for $3-4 \mathrm{~h}$ at room temperature. Finally, the PLGA-PTX-CuTCPP (P@PTX-CuTCPP) was obtained after centrifugation at 12000 rpm for $5 \mathrm{~min}$. The preparation of PLGA-PTX (P@PTX) was performed similar to the above process except that CUTCPP solution was replaced by deionized water at same volume. The similar method also was applied to the synthesis of PLGA-CuTCPP (P@CuTCPP) except removing PTX from stock solution. The P@PTX-CuTCPP was further coupled to tLyP1-PEl. Briefly, the carboxylic groups on the surfaces of PLGA was activated by adding into 2-(N-morpholino) ethanesulfonic acid (MES) buffer solution (0.05 $\mathrm{mM}, \mathrm{pH}$ 5.5) containing with of N-hydroxysuccinimide (NHS) and N-(3-dimethylaminopropyl)-N'ethylcarbodiimide hydrochloride (EDC) and the mixture was incubated in a shaker for $2 \mathrm{~h}$ at room temperature to activate the terminal carboxyl groups. Secondly, MES buffer solution $(0.05 \mathrm{mM}, \mathrm{pH} 8.0)$ was used to dissolve the mixture again after centrifugation. Thirdly, tLyP1-PEI (10 mg) was added and allowed to react for another $12 \mathrm{~h}$. Finally, the resulting tLyP-1-PEI-PLGA-PTX-CuTCPP (TPP@PTXCuTCPP) was purified by repeated washing 3 times with deionized water to remove unreacted materials. The preparation PEI-PLGA-PTX-CUTCPP (PP@PTX-CuTCPP) was prepared similar to the above method excepting that tLyP1-PEI was replaced by PEI (10 mg).

\subsection{Characterization of TPP@PTX-CuTCPP}

The morphology of TPP@PTX-CuTCPP was observed by scanning electron microscopy (SEM, Hitachi S$3400 \mathrm{~N}$, Japan) and transmission electron microscopy (TEM, Hitachi H-7600, Japan). The zeta potentials and size distributions were determined by a dynamic light scattering (DLS, Malvern, NanoZS, UK). The absorption spectrum of TPP@PTX-CuTCPP, tLy-1-PEI-PLGA-PTX-CuTCPP (TPP@PTX), and CuTCPP aqueous solutions were measured by UV-vis spectroscopy (Shimadzu, UV-3600, Japan) to observe the 
presence of CuTCPP in the NPs. The standard curve of free CuTCPP was calculated to evaluate the amount of CuTCPP encapsulated into PLGA. The entrapment efficiency and content of CuTCPP were calculated by Equations (1).

(1) CuTCPP entrapment efficiency $(\%)=\frac{\text { Mass of total CuTCPP }- \text { Mass of unentrapped CuTCPP }}{\text { Mass of total CuTCPP }} \times 100 \%$

The spectrum of PTX solutions at different concentrations, and TPP@PTX-CuTCPP (dissolved in DMSO and methyl alcohol $(\mathrm{v} / \mathrm{v})=1: 1)$ were measured by high performance liquid chromatography (HPLC," Waters, ZQ2000, China) system to calculate standard concentration curve for analyzing the amount of PTX in TPP@PTX-CuTCPP. The entrapment efficiency and content of PTX were calculated by Equations (2).

\section{(2) PTX entrapment efficiency (\%) $=\frac{\text { Mass of entrapped PTX }}{\text { Mass of total PTX }} \times 100 \%$}

\subsection{Drug Release}

Firstly, TPP@PTX-CuTCPP suspension was equivalently injected into two dialysis bags $(n=3$, MW cutoff $8,000-14,000 \mathrm{Da}$ ). The dialysis bags were placed in a plastic bottle containing $60 \mathrm{~mL}$ buffer solution containing with ethanol $(\mathrm{v} / \mathrm{v}=30 \%)$, sodium azide $(\mathrm{w} / \mathrm{v}=0.02 \%)$ and tween-80 $(\mathrm{v} / \mathrm{v}=0.1 \%)$ at different $\mathrm{pH}$ values $(\mathrm{pH}=6.3$ and 7.4$)$ under $150 \mathrm{rpm}$ stirring at room temperature. At every predetermined time point (preinjection and $1,3,7,12,18$, and $24 \mathrm{~h}$ ), $1 \mathrm{~mL}$ of buffer solution in the plastic bottle were collected for measurement by DLS and HPLC system and replaced by $1 \mathrm{~mL}$ of fresh buffer solution into the plastic bottle at the same time. Finally, variation of size distributions and cumulative release ratio in two groups were calculated.

\subsection{Cell Culture and Tumor-bearing Animal Model Establishment}

MCF-7/Taxol cells were maintained in RPMI-1640 medium (HyClone, USA) supplemented with $20 \%$ fetal bovine serum (FBS, Bioind, Israel), $100 \mathrm{U} / \mathrm{mL}$ penicillinand-streptomycin and $0.5 \mu \mathrm{g} \mathrm{mL}^{-1} \mathrm{PTX}$ solution. MCF-7 cells were maintained in 1640 medium supplemented with $10 \% \mathrm{FBS}$ and $100 \mathrm{U} \mathrm{mL}^{-1}$ penicillinandstreptomycin solution. HUVECs were maintained in RPMI-1640 medium supplemented with $10 \%$ FBS and $100 \mathrm{U} \mathrm{mL}^{-1}$ penicillinand-streptomycin solution. The cells were maintained in an incubator containing $5 \%$ $\mathrm{CO}_{2}$ atmosphere at $37^{\circ} \mathrm{C}$. All female BALB/c nude mice (16 to $20 \mathrm{~g}, 4$ to 6 weeks) were bred in humidity conditions between 18 to $20^{\circ} \mathrm{C}$. Food and water were free to provide. All protocols were approved by the Institutional Animal Care and Use Committee of Chongqing Medical University. To establish the MCF7/Taxol tumor models, $4 \times 10^{6} \mathrm{MCF}-7 /$ Taxol cells suspended in PBS solution $(100 \mu \mathrm{L})$ were subcutaneously injected into the right flank of each nude mouse. To establish an artificial bilateral tumor model, $2 \times 10^{6} 4 \mathrm{~T} 1$ cells suspended in PBS solution $(100 \mu \mathrm{L})$ were orthotopically inoculated into the right breast fat pad of the thirty BALB/c mice as primary tumor. 7 days later, $4 \mathrm{~T} 1$ cells were injected into the 
left breast fat pad as distant tumor. The tumor-bearing mice were used for additional in vivo experiments and the volume of tumors were calculated by the following equation: [ $\pi / 6 \times$ length $\left.\times(\text { width })^{2}\right]$.

\subsection{Cell Targeting and Tumor Spheroid Penetrating Efficiency}

MCF-7/Taxol and Human umbilical vein endothelia cells (HUVECs) were seeded into a laser confocal cellculture dish at a density of $2 \times 10^{5}$ cells per well respectively. After $24 \mathrm{~h}$ incubation, the cell culture medium was replaced with the medium containing Dil-labeled $\left(\lambda_{\text {excitation }} / \lambda_{\text {emission }}=549 \mathrm{~nm} / 565 \mathrm{~nm}\right)$ TPP@PTX-CuTCPP or PP@PTX-CuTCPP for 0.5, 1, and 2 h, respectively. For the qualitative study, the cells were washed three times with PBS and fixed with $4 \%$ paraformaldehyde for $15 \mathrm{~min}$ at room temperature. The cell nuclei were then stained with DAPI for $10 \mathrm{~min}$. Finally, the fluorescence images of the treated cells were acquired by confocal laser scanning microscopy (CLSM, Nikon, A1, Japan). In addition, the quantitative intracellular uptake of TPP@PTX-CuTCPP or PP@PTX-CuTCPP for MCF7/Taxol and HUVEC cells at different intervals were analyzed by flow cytometry (Becton Dickinson, FACS Vantage, USA). Three dimensional spheroids of MCF-7/Taxol cells were established by seeding cells in ultra-low adherent plates. Briefly, $1 \times 10^{5} \mathrm{MCF}-7 /$ Taxol cells per well were seeded in 6-well ultra-low adherent plates for 10 days formation. The spheroids were then coincubated with Dil-labeled TPP@PTXCuTCPP or PP@PTX-CuTCPP for $6 \mathrm{~h}$ and send for CLSM observation after washing twice with PBS, respectively. Multiple level scans for three dimensional tumor spheroids were done at $2 \mu \mathrm{m}$ intervals to measure penetration depth.

\subsection{In Vitro and In Vivo PAl:}

To determine the photoacoustic imaging (PAI) performance of TPP@PTX-CuTCPP, a 3\% agarose gel phantom with a hole was initially constructed. The PAl images were obtained by a photoacoustic imaging system (Vevo LAZR, Canada). TPP@PTX-CuTCPP $\left(200 \mu \mathrm{L}, 250 \mu \mathrm{g} \mathrm{mL}^{-1}\right)$ was used for full wavelengths scanning to detect the maximum absorbance. Varied CuTCPP concentrations of TPP@PTX-CuTCPP $\left(125,150,175,200,225,250 \mu \mathrm{g} \mathrm{mL}^{-1}\right)$ dissolved in deionized water were added to acquire the corresponding photoacoustic images by Vevo LAZR software at the excitation in $690 \mathrm{~nm}$. The quantified photoacoustic value within tumor region of each image was then analyzed. For in vivo PAI, MCF-7/Taxol tumor-bearing mice were intravenously injected with TPP@PTX-CuTCPP saline solution. Then, the PAI images were obtained at various time intervals (preinjection and, 1, 2, 4, 6, 12 and $24 \mathrm{~h}$ postinjection) and the photoacoustic intensity in the tumor regions was measured.

\subsection{Intraellular Uptake and Endosomal escape}

The cellular uptake of Dil-labeled PP@PTX, P@PTX, and free FTIC-PTX $\left(\lambda_{\text {excitation }} / \lambda_{\text {emission }}=488 \mathrm{~nm} /\right.$ $525 \mathrm{~nm}$ ) was visualized by CLSM. MCF-7/Taxol cells were seeded into confocal dishes at a density of $2 \times$ $10^{5}$ cells per well. After $24 \mathrm{~h}$ incubation, the cell culture medium was replaced with the serum-free medium containing Dil-labeled PEI-PLGA-PTX (PP@PTX), P@PTX or free FITC-PTX for $6 \mathrm{~h}$ of incubation. The cells were washed with PBS three times after incubation and treated with serum-free cell culture 
medium for another $2 \mathrm{~h}, 4 \mathrm{~h}, 6 \mathrm{~h}, 8 \mathrm{~h}, 10 \mathrm{~h}, 12 \mathrm{~h}$ respectively. At predetermined time point, cells were rinsed to remove effluent PTX and then lysed with RAPI cell lysis buffer (Beyotime, China). The fluorescence intensities of intracellular PTX $(n=3)$ were calculated by using a microplate reader (Bio-Tek Instrument Inc. USA) and observed by CLSM. To visualize the endo/lysosomal escape properties of NPs, the endo/lysosomes were stained to observe the colocalization of intracellular PP@PTX and P@PTX. After6 h coincubation with the medium containing Dil-labeled PP@PTX and P@PTX, the culture medium was replaced with fresh medium containing Lysotracker $\left(\lambda_{\text {excitation }} / \lambda_{\text {emission }}=504 \mathrm{~nm} / 511 \mathrm{~nm}\right)$ and incubated for another $0.5 \mathrm{~h}$ to stain the endo/lysosomes. The cells were gently washed with PBS and stained with DAPI for 10 min. Finally, the fluorescence of NPs and cells were visualized with the CLSM. The co-localization of NPs and Lysotracker from five representative cell images was quantified with Fuji ImageJ software by calculating the Pearson's correlation coefficient. To investigate the endo/lysosomal membrane integrity, calcein was added in the presence of PP@PTX or P@PTX and coincubated with cells for $6 \mathrm{~h}$. After three PBS washing steps, the cells were captured by CLSM

\subsection{GSH Scavenging Effects in Solution and Cells}

The aliquots were prepared by mixing GSH $(200 \mu \mathrm{L}, 1 \mathrm{mM})$ with free CuTCPP at varied concentration $\left(12.5,25,50,100,200 \mu \mathrm{g} \mathrm{mL}^{-1}\right)$, P@CuTCPP (containing CuTCPP at same concentration), or deionized water $(n=3)$, respectively. The supernatant was then extracted from respective aliquot at predetermined time point ( $24 \mathrm{~h}$ ) for GSH assay kit and X-ray photoelectron spectroscopy (XPS, ThermoFisher, ESCALAB250, USA) according to the manufacturer's protocol. For detection of GSH in cells, Firstly, MCF$7 /$ Taxol cells were seeded in 6-well culture plates at a density of $2 \times 10^{5}$ cells. After $24 \mathrm{~h}$ incubation, cell culture medium were replaced with NP-free medium, medium containing PLGA with free BSO $\left(45 \mu \mathrm{gL} \mathrm{mL}^{-}\right.$

$\left.{ }^{1}\right)$, or P@CuTCPP containing concentration of CuTCPP $\left(30 \mu \mathrm{g} \mathrm{mL}^{-1}\right)$ for another $24 \mathrm{~h}$ incubation. The cells were centrifuged at $1000 \mathrm{rpm}$ for 5 min after counting and normalizing the numbers of cells in each group and disrupted by ultrasonic wave (200 w, $5 \mathrm{~min}$ ). The samples were centrifuged at $10000 \mathrm{rpm}$ for 10 min at $4^{\circ} \mathrm{C}$ to collect supernatant that was used for GSH assay.

\subsection{Cell Viability and Cellular Apoptosis Assay}

The MCF-7/Taxol and HUVEC cells were initially seeded in 96-well culture plates for $24 \mathrm{~h}$. Then, the culture medium was replaced with the medium containing TPP@CuTCPP at different concentrations based on PLGA $\left(100 \mu \mathrm{L}, 0.3125,0.675,1.25,2.5\right.$ and $\left.5 \mathrm{mg} \mathrm{mL}^{-1}\right)$. After $24 \mathrm{~h}$ of treatment, the CCK-8 assay was used to evaluate the viability of cells according to the manufacturer's protocol. The optical density (OD) at $450 \mathrm{~nm}$ was read with a microplate reader. To assess the anticancer efficacy in vitro, the MCF7/Taxol cells were treated by the following 8 groups $(n=3)$ : G1, control; G2, free PTX; G3, P@PTX; G4, P@PTX-CuTCPP; G5, PP@PTX; G6, TPP@PTX; G7, PP@PTX-CuTCPP; and G8, TPP@PTX-CuTCPP. Firstly, MCF-7/Taxol cells were seeded in 96 well plates at a density of $1 \times 10^{4}$ cells per well. $24 \mathrm{~h}$ later, fresh medium containing different NPs and free drugs with equivalent concentration of PTX $\left(50 \mu \mathrm{g} \mathrm{mL}^{-1}\right)$ were added to replace the previous medium and coincubated with the cells for another $24 \mathrm{~h}$. Finally, the cell viabilities were evaluated by CCK-8 assay. We performed cellular apoptosis assay by treating MCF- 
7/Taxol cells with the same above condition after incubation in 6-well culture plates for $24 \mathrm{~h}$. The cells were then collected in $200 \mu \mathrm{L}$ of binding buffer. PI and Annexin V-FITC were added for coincubation with the cells for $15 \mathrm{~min}$. The stained cells were analyzed using a flow cytometry.

\subsection{Living and Dead Cell Co-staining Experiment}

MCF-7/Taxol cells were seeded into a laser confocal cell-culture dish at a density of $2 \times 10^{5}$ cells per well for $24 \mathrm{~h}$ incubation, the cells were treated by the following eight groups and incubated for another $24 \mathrm{~h}$ : G1, control; G2, free PTX; G3, P@PTX; G4, P@PTX-CuTCPP; G5, PP@PTX; G6, TPP@PTX; G7, PP@PTXCuTCPP; and G8, TPP@PTX-CuTCPP with concentration of $50 \mu \mathrm{g} \mathrm{mL}^{-1}$ based on PTX. The therapy effects were detected by inverted fluorescence microscope (FL, Olympus IX53, Canada) after costaining with $40 \mathrm{nM}$ calcein $\mathrm{AM}$ and $4.5 \mu \mathrm{M}$ propidium iodide $(\mathrm{PI})$ in PBS buffer solution for 10 min at $37^{\circ} \mathrm{C}$. $\mathrm{PI}$ was excited at $633 \mathrm{~nm}$ and detected with a 660-710 nm bandpass filter. Calcein AM was excited at 488 $\mathrm{nm}$ and detected with a 500-550 $\mathrm{nm}$ bandpass filter.

\subsection{Anticancer Effects in Vivo}

Forty tumor-bearing mice were randomly divided into 8 groups $(n=5)$ : G1, Control; G2, Free PTX; G3, P@PTX; G4, P@PTX-CuTCPP; G5, PP@PTX; G6, TPP@PTX; G7, PP@PTX-CuTCPP; and G8, TPP@PTXCUTCPP. Corresponding formulations were injected into MCF-7/Taxol tumor-bearing mice intravenously at the equivalent dose of $2 \mathrm{mg} \mathrm{PTX} \mathrm{kg}^{-1}$ per mouse 4 times every $4 \mathrm{~d}$. The control group was injected with saline solution $(200 \mu \mathrm{L})$. The tumor-volume changes of each group were recorded and the weight of mice in different groups was monitored every $4 \mathrm{~d}$. Tumor volume changes were normalized using the relative tumor volumes (initial tumor volume $\left(\mathrm{V}_{0}\right)$ / current tumor volume $(\mathrm{V})$ ). On day 16 , all mice were sacrificed for tumor dissection. Targeted tumor tissue was stained with Proliferating cell nuclear antigen (PCNA), TdT-mediated dUTP nick-end labeling (TUNEL), and Hematoxylin-eosin (H\&E) for immunohischemical analysis. The main organs of the mice were excised and fixed for H\&E staining

\subsection{Anticancer Efficacy of Combined Chemotherapy and Immunotherapy.}

The bilateral tumor-bearing mice were then randomly divided into 6 groups $(n=5)$ : (1) control; $(2)$ control + PD-L1; (3) free PTX; (4) free PTX + PD-L1; (5) TPP@PTX-CuTCPP and (6) TPP@PTX-CuTCPP + PD-L1. The day was defined as day 0 when treatments were performed. Corresponding formulations were intratumorally injected into primary tumors (first tumors) of $4 \mathrm{~T} 1$ tumor-bearing mice at the equivalent dose of $1 \mathrm{mg} \mathrm{PTX} \mathrm{kg}^{-1}$ per mouse 3 times every $6 \mathrm{~d}$. The control group was injected with saline solution $(200 \mu \mathrm{L})$. Furthermore, anti-PD-L1 $\left(1.5 \mathrm{mg} \mathrm{kg}^{-1}\right.$ per mouse) were intravenously injected to the mice in control + PD-L1 group, free PTX + PD-L1 group and TPP@PTX-CuTCPP + PD-L1 group on day 1, 4, 7 and 11. To evaluate the antitumor efficacy of combined chemotherapy and anti-PD-L1, the tumor-volume changes of each group were recorded. Tumor volume changes on both sides were normalized using the relative tumor volumes (initial tumor volume $\left(\mathrm{V}_{0}\right)$ / current tumor volume $(\mathrm{V})$ ). On day 16 , all mice were sacrificed for tumor dissection Hematoxylin-eosin (H\&E) for immunohischemical analysis. Western blot 
was used to investigate the expression of P-gp in tumor cells. To assess the in vivo antitumor immune responses against distant tumors, the tumors were harvested and treated with $0.2 \%$ collagenase $D, 0.01 \%$ hyaluronidase and $0.002 \%$ DNase to produce a single-cell suspension. Flow cytometry was used to determine the proportions of tumor-infiltrating T cells (CD3 ${ }^{+}, \mathrm{CD}^{+}{ }^{+}$and $\mathrm{CD} 8^{+} \mathrm{T}$ cells). TNF-a, IFN- $\gamma$, IL-6, IL12 were examined by using ELISA kits according to the manufacturer's protocols. $\mathrm{CD} 3^{+}, \mathrm{CD} 4^{+}$, and $\mathrm{CD} 8^{+}$ $T$ cells in the distant tumor tissue section were stained for immunofluorescence.

\subsection{Fluorescence Imaging and Biodistribution in Vivo}

For the fluorescence imaging $(\mathrm{FL})$ and biodistribution assessment in vivo, MCF-7/Taxol tumor-bearing mice $(n=3)$ were injected with DIR-labiled $\left(\lambda_{\text {excitation }} / \lambda_{\text {emission }}=748 \mathrm{~nm} / 780 \mathrm{~nm}\right)$ TPP@PTX-CuTCPP or PP@PTX-CuTCPP solution via tail vein. FL images were acquired at preinjection and, 2, 6, and $24 \mathrm{~h}$ postinjection by using fluorescence imaging system (CRi Inc, USA), and the relative FL intensity of the tumor regions was recorded. The tumor tissues and major organs were harvested for ex vivo experiment through FL imaging.

\subsection{Biosatey Assay}

Twenty-four BALB/c nude mice were randomly divided into five groups $(n=4)$. Twenty mice were sacrificed at predetermined time points $(1,3,7,14$, and $28 \mathrm{~d})$ after postinjection of $200 \mu \mathrm{L}$ TPP@PTXCUTCPP at a dose of $5 \mathrm{mg} \mathrm{mL}^{-1}$ based on PLGA and four mice in control group after postinjection fo saline solution were sacrificed at 1 day. The blood samples were further collected for routine blood examination and biochemistry assay including L-lactate dehydrogenase, creatine kinase, alanine aminotransferase (ALT), aspartate transaminase (AST), total bilirubin, creatinine (CR), and urea nitrogen (BUN). The major organs of the mice were harvested and fixed with $4 \%$ polyoxymethylene and stained with H\&E for histological analysis.

\subsection{Statistical Analysis}

All statistical analyses were analyzed by SPSS software 20.0 (Chicago, USA). Quantitative data were presented as the mean \pm standard deviation (SD). The significance of the data was analyzed according to the one-way ANOVA tests and Student's t-test. The level of significance in the statistical analyses was defined as $* p<0.05, * * p<0.01$.

\section{Results And Discussion}

\subsection{Design, Synthesis, and Characterization of TPP@PTX-CuTCPP}

The synthesis process of TPP@PTX-CuTCPP NPs was accomplished in two steps, nanoplatform preparation and surface modification, as depicted in Scheme $\mathbf{S 1}$ andFigure 1A. In this work, PLGA acted as the nanocarrier for the coencapsulation of a hydrophilic molecule, i.e., CuTCPP, and a hydrophobic molecule i.e., PTX, via a typical double-emulsion process. We then confirmed the sound synthesis of tLyP- 
1 by mass spectrometry (ThermoFisher, TSQ Altis, American), which demonstrated a comparable molecular weight (833.97) to the short peptide (CGNKRTR) (Figure S1). Next, tLyP-1-PEI was prepared using the maleimide-thiol method as determined by nuclear magnetic resonance spectroscopy (NMR, AVANCE III, Bruker, Germany). NMR spectrum displayed peaks from 2.6 to $3.0 \mathrm{ppm}$ compared with the spectrum of PEl (peaks from 2.5 to $2.9 \mathrm{ppm}$ ) which were attributed to $\mathrm{CH}_{2}-\mathrm{N}$ stretch of $\mathrm{PEl}$, indicating the successful conjugation of tLyP-1 with PEI. In addition, tumor-targeting feature was preserved by modification process because the C-terminal sequence of tLyP-1 was not altered (Figure S2). The disappearance of characteristic peaks of tLyP-1 from HPLC also validated the synthesis of tLyP-1-PEI (Figure S3). Finally, tLyP-1-PEI was covalently bonded onto the surface of the nanosystem via a carbodiimide coupling reaction as characterized by Fourier transform infrared spectroscopy (ThermoFisher, iS50, American). The peaks at $3002 \mathrm{~cm}^{-1}$ and $2948 \mathrm{~cm}^{-1}$ in the tLyP-1-PEI-PLGA and PEIPLGA spectra can be attributed to the C-H stretch of PLGA. Evident characteristic peaks at $1649 \mathrm{~cm}^{-1}$ and $3426 \mathrm{~cm}^{-1}$ associated with the -CO-NH and - $\mathrm{NH}_{2}$ groups in tLyP-1-PEI-PLGA and PEI-PLGA provided direct evidence of the existence of amide bonds (Figure 1B)[42, 43]. Moreover, the zeta potentials of the NPs modified with tLyP-1-PEI or only PEI were increased to a positive value, which was attributed to the positive charge from either the tLyP-1 peptide or PEl compared with the pristine NPs, according to DLS measurements (Figure 1C). This also suggested the successful binding of tLyP-1-PEI or PEI to the NPs. The tLyP-1-PEl functionalized PLGA shell endowed the multitasking nanoagent with endo/lysosomal escape and tumor homing-penetrating behavior providing the NPs with outstanding intracellular drug retention and allowing their rapid crossing of the tumor endothelial barrier. Moreover, depleting highleveled GSH followed after the codelivery of CuTCPP to recover PTX in MCF-7/Taxol cells. The morphology of the obtained TPP@PTX-CuTCPP NPs was then revealed by SEM and TEM. These NPs displayed well-defined appearance and monodispersed distribution with a size of approximately $300 \mathrm{~nm}$ size, which was comparable to $354.8 \mathrm{~nm}$ as measured by DLS (Figure 1D and 1E). In addition, That TPP@PTX-CuTCPP NPs had a polydispersity index (PDI) of 0.14 in deionized water indicated their highly homogeneity, which matched well with the results of fluorescence and optical imaging (Figure S4). In particular, TPP@PTX-CuTCPP exhibited good stability in various physiological solutions such as water, PBS, and RPMI-1640 culture medium containing FBS (Figure S5). Afterwards, UV-vis spectroscopy and HPLC were performed to confirm the successful loading and calculate the entrapment efficiency of the cargoes via their concentration and related absorbance (Figure 1F). The amount of CuTCPP loaded in TPP@PTX-CuTCPP was quantified by UV-vis spectroscopy, which demonstrated a characteristic absorption peak at the wavelength of $415 \mathrm{~nm}$, which was slightly redshifting from $412 \mathrm{~nm}$. However, no characteristic absorption peak was observed in NPs without encapsulation of CuTCPP (Figure 1G). Digital photographs also validated the successful encapsulation of CuTCPP due to the appearance of the aqueous solution changing from white to pink after CuTCPP loading (Figure S6). The CuTCPP entrapment efficiency were determined to be $27.1 \%$ by UV-vis spectroscopy. Additionally, the entrapment efficiency of PTX were approximately $70.1 \%$ with a characteristic band at $227 \mathrm{~nm}$ by HPLC (Figure S7 and S8).

\subsection{Intracellular Uptake and Endo/lysosomal Escape Efficiency}


Considering that MDR is mostly understood as the exportation of drugs by efflux pumps, we hypothesized that high-efficiency intracellular uptake and preservation of NPs were central to improving the clinical outcomes of chemotherapy. Thus, the properties of PEl-modified NPs to assist chemotherapeutic agents in overcoming MDR through escape from endo/lysosomal sequestration and hydrolytic degradation were further studied. To observe intracellular drug retention, MCF-7/Taxol cells were first treated with NPs with PEI modification, NPs without PEI modification or free FITC-PTX, which was then replaced with a NP-free solution for follow-up observations. As evaluated by CLSM images (Figure 2A), extensive NPs emitting red fluorescence were visible in the cytoplasm in MCF-7/Taxol cells in the PP@PTX group, than in the P@PTX and free FTIC-PTX groups, where the PTX level decreased rapidly in the MCF-7/Taxol cells. This outcome suggested that there was a significantly slower drugs efflux rate when the cells were coincubated with PP@PTX. The fluorescence intensities further revealed that concentrations of intracellular PTX in MCF-7/Taxol cells treated with free FITC-PTX and P@PTX were only $38.0 \%$ and $53.3 \%$, suggesting apparent drug efflux via P-gp. In contrast, the retention ratio of PTX increased significantly when the cells were treated with PP@PTX, reaching 82.0 \% PTX retention in MCF$7 /$ Taxol cells. The foregoing results collectively indicated that PTX efficiently accumulated and retained in drug-resistant cells with the assistance of PEl-modified nanoagents (Figure 2B and S9). To investigate the mechanism of drug retention enhancement that was probably mediated by endo/lysosomal escape, CLSM was subsequently employed to observe the colocalization of NPs and endo/lysosomes in MCF7/Taxol cells after coincubation with PP@PTX and P@PTX. As depicted in Figure 2C, most P@PTX NPs were trapped inside the endosome or lysosome which showed yellow fluorescence (due to the overlap of green and red dots). In contrast, distinct red and green signals were observed when analyzing the PP@PTX group, which indicated the successful endo/lysosomal escape of the NPs. Moreover, the corresponding Pearson's correlation coefficient (PCC) was determined to be markedly lower in the PP@PTX group than that in the P@PTX group, again suggesting a low degree colocalization of PP@PTX with endo/lysosomes (Figure 2D)[44, 45]. Calcein, a membrane-impermeable fluorophore, to assess the integrity of endo/lysosomal membrane, was also used which displays punctuated fluorescence patterns when it is entrapped in endo/lysosomes. However, a spread and bright fluorescence was detected after conincubation with PP@PTX, implying endosomal/lysosomal membrane was disrupted and calcein escaped. Furthermore, the biological TEM images of the cells before and after incubation with PP@PTX (preincubation and 3 , and $6 \mathrm{~h}$ after incubation) were used to visualize the process of endo/lysosomal destruction. The oval shaped endosomes or lysosomes with were charged with electron-dense material before treatment with the NPs. Some of these organelles displayed morphological changes after coincubation with PP@PTX, becoming swollen with partial membrane rupture as depicted in Figure 2E and S10[46]. These results proved the endo/lysosomal destruction capability owing to the proton-sponge effect of the PEI-modified NPs, thereby enhancing the release of the cargoes. In light of the excellent intracellular accumulation and endosomal escape capability of the PEI-modified NPs, we hypothesized that PP@PTX allowed more anticancer drugs to be retained in the MDR cells to increase the cytosolic concentration of PTX, and thus mitigating drug resistance caused by efflux pumps[47]. The cytotoxicities of PP@PTX and P@PTX with different PTX concentrations in MCF-7/Taxol cells was then studied. PP@PTX was first immersed in PBS at pH 6.3 for $2 \mathrm{~h}$ to simulate the acidity of the TME. After 
coincubation with different formulations (pretreated PP@PTX, P@PTX, and PP@PTX) for 24 h, the viability of the MCF-7/Taxol cells was assessed by using the CCK-8 assay. Notably, the decrease in cell viability depended on the increased drug concentration, which decreased more sharply in the $\mathrm{pH}$-treated PP@PTX group compared with the other groups. This results was speculated to be due to the fact that PEI-modified NPs more readily trigger destabilization of the endosomal membrane by absorbing numerous protons in the lower $\mathrm{pH}$ environment, leading to better intracellular retention and cytotoxic effects to reverse MDR in vitro (Figure 2F)[48].

\subsection{In Vitro and Vivo of Targeting and Penetrating Abilities}

To demonstrate that tLyP-1 could enhance the targeting ability of the nanoplatform, the intracellular behavior of TPP@PTX-CuTCPP was visualized by CLSM. As shown in Figure 3A, increased Dil-labeled TPP@PTX-CuTCPP emitting red fluorescence aggregated around the cytomembrane of the MCF-7/Taxol cells that overexpressed NRP- 1 after coincubation with monolayer cells for $0.5,1$, and $2 \mathrm{~h}$. However, fewer NPs gathered around the cells in the Dil-labeled PP@PTX-CuTCPP group. The role of tLyP-1 in guiding TPP@PTX-CuTCPP to NRP-1 receptor-rich tumor cells was verified by incubating with NRP-1 receptornegative HUVECs as a control. The results demonstrated that the number of aggregated TPP@PTXCuTCPP decreased markedly in HUVECs compared with MCF-7/Taxol cells. The flow cytometry results, which revealed stronger fluorescence in the MCF-7/Taxol cells after coincubation with TPP@PTXCuTCPP were, in accordance with the CLSM images (Figure 3B). To study the penetration mechanism of NPs functionalized by THPP, a three-dimensional tumor spheroid model was successfully established in this study which can better simulate the TME of solid tumor due to the high cell density, increased interstitial pressure, and heterogeneous tumor perfusion in the spheroid model. After coincubation with Dil-labeled TPP@PTX-CuTCPP or PP@PTX-CuTCPP for 6 h, we found that the red fluorescence from TPP@PTX-CuTCPP was more evidently distributed in the tumor spheroids more compared with PP@PTXCuTCPP (Figure 3C and S11 and S12). Furthermore, tLyP-1 enabled these versatile nanosystems to distribute throughout the whole spheroid; however, PP@PTX-CuTCPP adhered to only the margin of the tumor spheroid via three-dimensional reconstruction. Quantitative analysis revealed that the penetration depth of TPP@PTX-CuTCPP $(21.30 \mu \mathrm{m})$ reached to the core of tumor spheroid was, which was 2.82 -fold greater than that of PP@PTX-CuTCPP with a penetration depth of only $7.55 \mu \mathrm{m}$ (Figure 3D, 3E and 3F). The above results collectively indicated that the tLyP-1 peptide could facilitate the transportation of the NPs into the core of tumor spheroids possibly favoring the MDR reversal. Additionally, we used MCF7/Taxol tumor-bearing mice to validate the in vivo targeting ability via FL imaging and quantitative analysis. The fluorescence intensity at the tumor location in the TPP@PTX-CuTCPP group was significantly stronger than that in the PP@PTX-CuTCPP group at the predetermined time intervals. The FL signal peaked at $6 \mathrm{~h}$ after the administration of DiR-labeled TPP@PTX-CuTCPP, which also exhibited relatively longer retention in the tumor region than the nontargeted group (Figure $\mathbf{3 G}$ and $\mathbf{3 H}$ ). Then, the tumors and major organs (heart, liver, spleen, lung, and kidney) were next harvested to determine the ex vivo biodistribution of the NPs $24 \mathrm{~h}$ postinjection. The results revealed that both two types of NPs were accmulated in the liver and spleen due to phagocytosis by the reticuloendothelial system (RES). However, greater accumulation of the NPs in tumor tissue was found in TPP@PTX-CuTCPP group as shown in 
Figure3I and S13. Meanwhile, the ultrathin tumor frozen sections were prepared for CLSM analyses. The TPP@PTX-CuTCPP group contained more red dots in tumor tissue compared with PP@PTX-CuTCPP

(Figure S14). These above results demonstrated again the desirable targeting feature of tLyP-1. In particular, we found that PP@PTX-CuTCPP NPs mainly gathered in microvessels, which were stained by CD31, with little extravasation. However, TPP@PTX-CuTCPP NPs spread into the extravascular tumor tissue and reached into a deeper area (Figure S15 and S16). This evidence revealed firmly that thyP-1 functionalized NPs with active tumor-penetrating property are promising for anticancer drug carriage.

\subsection{Scavenging Effect of GSH and PAl in Vitro and in Vivo}

It is anticipated that $\mathrm{Cu}^{2+}$-based nanoagent can cause an imbalance in the the $\mathrm{GSH}$-involved redox metabolism equilibrium, further relieveing MDR by reshaping the TME. The proposed mechanism was first studied in GSH solution and tested by 5,5'-dithiobis-2-nitrobenzoic acid (DTNB), a tracker for relative intracellular GSH levels by monitoring the variation in UV-vis absorbance intensity of the intermediate product at $405 \mathrm{~nm}$, after mixing with NPs delivering CuTCPP, free CuTCPP, and deionized water at varying concentrations for $24 \mathrm{~h}$. As depicted in Figure 4A, there was comparative GSH-depletion efficiency from the free CuTCPP and P@CuTCPP groups containing equivalent dose of CuTCPP, which suggested the nanosystem comprising CuTCPP inherited the potent GSH scavenging capability of CuTCPP. In addition, the valence state of $\mathrm{Cu}$ was determined by XPS, which enables surface analysis with a quantitative accuracy of $90-95 \%[49,50]$. The binding energies of Cu2 $\mathrm{p}_{1 / 2}(954.48 \mathrm{eV})$ and $\mathrm{Cu} 2 \mathrm{p}_{3 / 2}(934.98 \mathrm{eV})$ in CuTCPP, which can be assigned to $\mathrm{Cu}^{2+}$ peaks, were observed before treatment with $\mathrm{GSH}$. The satellite peak at $942.98 \mathrm{eV}$ between $\mathrm{Cu} 2 \mathrm{p}_{3 / 2}$ and $\mathrm{Cu} 2 \mathrm{p}_{1 / 2}$ in the paramagnetic chemical state also provided credible evidence for the presence of the $\mathrm{Cu}^{2+}$ valence state. Interestingly, the binding energies of $\mathrm{Cu} 2 \mathrm{p}_{3 / 2}$ and Cu2 $\mathrm{p}_{1 / 2}$ shifted slightly to $953.88 \mathrm{eV}$ and $934.68 \mathrm{eV}$, respectively, and decrease in the original satellite peak were found after treating GSH solution. Additionally, we further analyzed the sample by comparing the integrated peak areas. The atomic percentage of $\mathrm{Cu}^{2+}$ decreased from $96.40 \%$ to $30.66 \%$ after treatment with GSH. These results firmly suggested the partial reduction in the copper valence state from +2 to +1 during the reaction (Figure 4B and S17) [51, 52]. Subsequently, MCF-7/Taxol cells were used to detect intracellular GSH levels after coincubation with P@CuTCPP, and PLGA in combination with Lbuthionine sulfoximine (L-BSO), an inhibitor of GSH synthesis. The results revealed that even though LBSO inhibited the synthesis of intracellular GSH most remarkably, P@CuTCPP also exhibited moderate GSH-depletion performance among the three groups (Table S1). Inspired by the considerable GSH depletion by CUTCPP, we assessed the cytotoxicity of PTX in the presence and absence of CuTCPP to verify whether the GSH scavenging effect sensitized the chemotherapy against MDR cells. Notably, the viability of MCF7/Taxol cells treated with P@PTX-CuTCPP or P@PTX combined with nontoxic dose of LBSO was significantly lower than that of cells treated with P@PTX only as shown in Figure 4C. Light absorption in the NIR region is a prerequisite for NIR-induced imaging. To investigate the potential of TPP@PTX-CuTCPP as a PAl contrast agent for tumor monitoring, an in vitro gel experiment was initially performed. An excitation wavelength of $690 \mathrm{~nm}$ was selected as the optimal wavelength for enhanced PAl in the following experiments after full spectrum scanning in photoacoustic system (Figure 4D). The 
results showed that the photoacoustic intensities strengthened after increasing the CuTCPP concentration from 125 to $250 \mu \mathrm{g} \mathrm{mL}^{-1}$. Quantitative analysis revealed that photoacoustic intensity of the NPs was concentration-dependent with a good linear relationship $\left(R^{2}=0.99\right)$ corresponding to contrastenhanced imaging, which indicated that the imaging features of TPP@PTX-CuTCPP were triggered by laser irradiation (Figure 4E). Subsequently, MCF-7/Taxol tumor-bearing mice were used to confirm PAI capability after postinjection of TPP@PTX-CuTCPP or PP@PTX-CuTCPP solution. The photoacoustic images were then obtained at predetermined time point (preinjection, and 1, 2, 6, 12, and $24 \mathrm{~h}$ postinjection), and corresponding quantitative photoacoustic intensity analysis was performed. The results showed that there were negligible photoacoustic signals at the tumor region in both group before NP injection. After injection with TPP@PTX-CuTCPP, the photoacoustic signal at the tumor region gradually increased and reached a peak value approximately $6 \mathrm{~h}$ postinjection which was dramatically stronger than that observed in the PP@PTX-CuTCPP group. With increasing time after post-injection, the tumor photoacoustic value started to decrease attributing to the clearance of NPs; nevertheless, the signal in the TPP@PTX-CuTCPP group was still significantly stronger than that observed in the PP@PTXCuTCPP group owing to the targeting behavior of tLyP-1 (Figure 4F and 4G). These results confirmed that the TPP@PTX-CuTCPP could effectively accumulate in tumor tissue and serve as contrast-enhanced agent for PAl.

\subsection{Cytotoxicity and Anticancer Effects in Vitro}

The release process of PTX from the NPs were quantitatively investigated by soaking the samples in buffer solution at different $\mathrm{pH}$ values $(\mathrm{pH}=6.3$ and 7.4) to simulate the intracellular environments. Approximately 68.1 \% of the loaded PTX was released from TPP@PTX-CuTCPP immersed at pH 6.3 after $24 \mathrm{~h}$, higher than $38.3 \%$ released from these NPs in solution at physiologic $\mathrm{pH}$ (7.4) (Figure 5A). This may be explained by the responsiveness of the NPs to a weakly acidic environment in which the PLGA shell layer gradually disassembles, triggering the high-efficiency release of PTX from the delivery system[53]. Interestingly, time-dependent changes in NPs size at varying time intervals were observed by DLS and TEM. An increasingly smaller size of NPs was detected over time, as presented in Figure 5A and S18, which corresponded to the PTX release ratio. Due to the impressive drug release behavior of the NPs, the in vitro cytotoxic and anticancer effects were next investigated. The IC50 values of PTX against the parental cells (MCF-7) and drug-resistant cells (MCF-7/Taxol) were initially determined using the CCK-8 assay. An 18.78-fold higher $\mathrm{IC}_{50}$ value was observed in MCF-7/Taxol cells compared with that in MCF-7 cells, suggesting the successful establishment of a PTX-resistant cell line. (Figure 5B and 5C) Further, the cytotoxicity of TPP@CuTCPP (PTX-free) was evaluated in MCF-7/Taxol cells and HUVECs to assess the biosafety of the drug-delivery vehicle and verify that the anticancer effects originate from PTX. TPP@CuTCPP exerted only a negligible effect on the survival of MCF-7/Taxol cells and HUVECs even at PLGA concentrations as high as $5 \mathrm{mg} \mathrm{mL}^{-1}$, as shown in Figure 5D, displaying favorable low toxicity and biocompatibility as a drug carrier for further application. To study the anticancer effects of the various NPs after carrying PTX, the viabilities of MCF-7/Taxol cells treated with the following eight compounds for 24 h were evaluated: G1, control; G2, free PTX; G3, P@PTX; G4, P@PTX-CuTCPP; G5, PP@PTX; G6, 
TPP@PTX; G7, PP@PTX-CuTCPP; and G8, TPP@PTX-CuTCPP. As shown in Figure 5E, limited cytotoxicity was detected in the group of free PTX and P@PTX, which could be ascribe to the drug resistance speciality from MCF-7/Taxol cells. When PEI, CuTCPP, or tLyP-1 was added to the nanosystem, the cell viability markedly decreased to different degrees. Of note, TPP@PTX-CuTCPP exhibited significantly greater cytotoxicity than the other treatments, demonstrating the best anticancer efficacy. The coincubation of MCF-7/Taxol cells with various concentrations of TPP@PTX-CuTCPP was then investigated. Cell viability decreased with increasing TPP@PTX-CuTCPP concentrations, showing that the therapeutic effect was concentration-dependent (Figure S19). Flow cytometry was performed to quantitatively analyze cell apoptosis induced by the NPs. The determined percentage of apoptotic and live cells was similar to that found in the CCK-8 assay (Figure 5F and 5G). Moreover, to visually evaluate the synergistic effects of the various formulations, we stained the cells with PI and calcein AM to determine the dead and live cells, which also confirmed that TPP@PTX-CuTCPP induced maximum cell death (Figure $\mathbf{5 H}$ ). To prove that combined reversing strategies was synergism instead of an additive effect, the combination indexes (Cls) of different nanofomulations were calculated by Chou-Talalay method[54]. As shown in Table S2, nanoformulations equipping one or two strategies showed moderate synergism to PTX, notably, Cl of nanoformulation with triple strategies (TPP@PTX-CuTCPP) was 0.201, which indicated the strong synergism. These results jointly indicated that the multifunctional NPs possessing tumor homing-penetrating, endo/lysosomal escape and GSH depletion capabilities can synergistically enhance the anticancer efficacy of PTX for the reversal of MDR.

\subsection{Anticancer Therapy in Vivo}

The chemotherapeutic efficacy in vivo was evaluated by monitoring tumor growth and measuring the changes in tumor volume for 16 days in MCF7/Taxol tumor-bearing mice (Figure 6A). The tumor-bearing mice were randomly divided into eight groups for different treatments $(n=5): G 1$, control; $G 2$, free PTX; G3, P@PTX; G4, P@PTX-CuTCPP; G5, PP@PTX; G6, TPP@PTXP; G7, PP@PTX-CuTCPP; and G8, TPP@PTX-CuTCPP. As shown in Figure 6C and 6D, the mice treated with various compounds slowed down tumor growth to different degrees during 16 days of observations and had most superior antitumor effects after treatment with G8. In addition, it was noteworthy that G8 was the only group that not merely suppressed the rate of the tumor growth, but also shrank the tumor volume at the end of treatment

(Figure 6E). Furthermore, tumor inhibition rate dramatically increased by $91.4 \%$ in the G8 compared with that in control group at $16 \mathrm{~d}$ postinjection, suggesting that there was an almost tumor eradication (Figure 6B). In contrast, limited tumor inhibition was observed in the control group and the G2, which was similar to the results found in vitro test. Thus, we speculated that the "combo" nanoagent acting on different mechanisms of drug resistance through multiple synergistic strategies would provide best efficacy for the reversal of MDR. PCNA, TUNEL, and H\&E staining of the tumor sections further was conducted to confirm the chemotherapeutic efficacy of the multifunctional NPs. H\&E staining showed that severe apoptosis and necrosis were found in the G8, while only moderate damage appeared in the G7, G6, and G5. The PCNA and TUNEL assays had the similar trends. Representative apoptosis-positive cells are indicated by dark-brown nuclei in the TUNEL assay. Of note, the most positive index was observed in the group receiving TPP@PTX-CuTCPP, where the tumors were almost eliminated without relapse at the end of 
treatment period. PCNA immunochemical staining of the tumors showing the in vivo proliferative activities was consistent with the results of the TUNEL assay where proliferative cells were stained into brown (Figure 7A). The results of TUNEL and PCNA assays collectively demonstrated that TPP@PTXCuTCPP has greatest MDR reversal efficacy than a single strategy. Additionally, H\&E staining of the major organs (including the heart, liver, spleen, lungs, and kidneys) showed no pathological damage or inflammatory lesion during the in vivo treatments in all groups (Figure S20).

\subsection{Biosafety Assay of TPP@PTX-CuTCPP in Vivo}

The in vivo biosafety of the TPP@PTX-CuTCPP in both short and long terms $(1 \mathrm{~d}, 3 \mathrm{~d}, 7 \mathrm{~d}, 14 \mathrm{~d}$, and $28 \mathrm{~d}$ of postinjection) was evaluated by histopathological and hematological analyses. H\&E staining of the main organs indicated no histopathological changes or an abnormal inflammatory response in the all groups compared to the control group (Figure 7B). Meanwhile, blood biochemical indexes showed no evident increase in the levels of indexes compared with the control group, indicating that the chemotherapy based on TPP@PTX-CuTCPP have good biocompatibility, which would make it possible for future clinical translation (Figure 7C). The body weight of the mice was also monitored during the period of therapy as shown in Figure S21. There were negligible differences found in all groups, suggesting that the anticancer effects carried by nanosized delivery system induce undetectable side effects to mice at the tested dose.

\subsection{Combined Immunochemotherapy and Immune Responses in Vivo}

To assess the in Vivo anticancer efficacy in combination of multifunctional nanoagents with checkpoint blockade therapy and the immune reaction after treating with anti-PD-L1, bilateral mimic tumor models (primary and distant) in BALB/c mice were established (Figure 8A). In following experiments, antitumor efficacy initiated by immunochemotherapy was firstly investigated. For the primary tumors, both TPP@PTX-CuTCPP and TPP@PTX-CuTCPP + anti-PD-L1 inhibited tumor growth within 16 days posttreatment notably, whereas, free PTX or free PTX plus anti-PD-L1 showed no appreciable effect on tumor growth (Figure 8B and E). Moreover, TPP@PTX-CuTCPP plus anti-PD-L1 exhibited superior antitumor efficacy, which probably derived from synergistic effect of immunochemotherapy. For the distant tumors, due to the activation of antitumor immunity from anti-PD-L1 treatment, tumor growths in the control + PD-L1, free + PD-L1 and TPP@PTX-CuTCPP plus anti-PD-L1 group were partly suppressed. Massive apoptosis and necrosis stained by H\&E was found in tumor area which was consistent with appearance of tumor growth as revealed in Figure $\mathbf{8 C}, \mathbf{F}$ and $\mathbf{S 2 2}$. However, there is no significant inhibition effect on the distant tumors in the group without anti-PD-L1 treatment. Subsequently, we evaluated systemic immunity elicited by anti-PD-L1. Helper T cells $\left(\mathrm{CD} 4^{+}\right)$and cytotoxic T lymphocytes $\left(\mathrm{CD} 8^{+}\right)$play important roles in the regulation of adaptive immunities and destruction of targeted cancer cells, therefore, the level of tumor infiltrating $\mathrm{T}$ cells in the mimic distant tumors were co-stained with $\mathrm{CD}^{+}, \mathrm{CD} 4^{+}$and $\mathrm{CD} 8^{+} \mathrm{T}$ cells for further flow cytometry analysis. The results showed that the proportion of $\mathrm{CD} 4^{+} \mathrm{T}$ cells in the TPP@PTX-CuTCPP + anti-PD-L1 group was $19.13 \%$, with a significant elevation compared with control group (1.18\%). On the other hand, the absolute percentage of $\mathrm{CD}^{+} \mathrm{T}$ cells in the combined 
immunotherapy group occupies $15.86 \%$ with an improvement by nearly 5.6 times in comparison with the control group (Figure S23). Interestingly, a similar trend was found in tumor tissue slices, in which greatly enriched $\mathrm{CD} 4^{+}$and $\mathrm{CD} 8^{+} \mathrm{T}$ cells in the distant tumor were observed by the immunofluorescence assay (Figure 8D). It was reported that Helper T cells could produce pro-inflammatory such as TNF-a, IL-6, and IFN- $\gamma$, all of which could enhance activation of innate immune response. ELISA assay was next employed to evaluate the secretion levels of TNF- $a$, IFN-y, IL- 6 and IL-12 in the serum from the mice in all groups. Consistent with the activation on tumor infiltrating $T$ cells, higher secretion levels mentioned above were observed in groups with anti-PD-L1 treatment, implying again a successful initiation of systemic immune response (Figure S24). To verify whether anti-PD-L1 could enhance cytotoxicity via regulating P-gp expression, western blot was performed to evaluate P-gp expression level in primary tumor. As depicted in Figure 8G, the P-gp expression level obviously decreased after treating with anti-PD-L1 in comparison to groups without anti-PD-L1 treatment, which may consequently lead to higher anticancer drugs accumulation in tumor cells.

\section{Conclusion}

In summary, we successfully developed a "combo" nanoscale medicine that exhibited a variety of synergistic strategies to enhance tumor chemotherapy aimed at overcoming MDR. tLyp-1-PEI functionalized NPs triggered rapidly tumor homing-penetration and endo/lysosomal escape effects after internalization by tumors. The PTX released from the cracked endo/lysosomes was expected to efficiently accumulate and be retained in MCF-7/Taxol cells to reverse MDR and inhibit the growth of tumors compared with the low efficacy of an equivalent dose of free PTX. On the other hand, by introducing CuTCPP into the nanosystem, upregulated GSH in tumor cells was scavenged, which might further render MDR cells more vulnerable to anticancer agents. Based on the NIR absorption from CuTCPP, TPP@PTX-CuTCPP with imaging potential is promising to serve as a PAl enhanced-contrast agent for theranostic guidance and visual monitoring in one-go. Additionally, immune reaction and P-gp inhibition were observed by blocking PD-1/PD-L1 interaction, indicating that checkpoint blockade therapy may contribute to combat chemotherapy resistance in breast cancer. Overall, the novel nanoagent in combination with immunotherapy may guarantee an attractive strategy for fighting against tumor drug resistance.

\section{Declarations}

\section{Acknowledgements}

The authors sincerely grateful for the financial supports from the National Natural Science Foundation of China (Grant No. 81873901, 81971633, 31630026, 81630047), Basic Research and Frontier Exploration Key Project of Chongqing Science and Technology Commission (Grant No. cstc2019jcyj-zdxmX0020), High-level Medical Reserved Personnel Training Project of Chongqing, Kuanren Talents Program of the Second Affiliated Hospital of Chongqing Medical University (KR2019G001), and Chongqing Science and Health Joint Medical Research Project-Young and Middle-aged High-level Talent Project (2020GDRC011). 


\section{Authors' contributions}

Weixi Jiang: Conceptualization, Methodology, Writing - original draft. Lei Su: Investigation. Meng Ao: Validation, Formal analysis. Xun Guo: Resources. Chen cheng: Conceptualization. Yuanli Luo: Data curation. Zhuoyan Xie: Visualization. Xingyue Wang: Data curation. Junrui Wang: Software, Formal analysis. Shuling Liu: Investigation. Yang Cao: Methodology. Pan Li: Conceptualization. Zhigang Wang: Project administration. Haitao Ran: Project administration. Zhiyi Zhou: Conceptualization, Supervision, Writing - review \& editing. Jianli Ren: Resources, Supervision, Writing - review \& editing.

\section{Availability of data and materials}

The data are available in the main manuscript, supplementary Information files are available from the corresponding author by request.

\section{Ethics approval and consent to participate}

All protocols were approved by the Institutional Animal Care and Use Committee of Chongqing Medical University

\section{Consent for publication}

All the authors have approved the manuscript and agree with submission to journal.

\section{Competing interests}

The authors declare no competing financial interest.

\section{Authors details}

${ }^{1}$ Department of Ultrasound, Chongqing Key Laboratory of Ultrasound Molecular Imaging, Chongqing Medical University Affiliated Second Hospital, Chongqing 400010, P. R. China. ${ }^{2}$ Department of Radiology, Chongqing Medical University Affiliated Second Hospital, Chongqing 400010, P. R. China. ${ }^{3}$ Department of General practice of Chongqing General Hospital, University of Chinese Academy of Sciences, Chongqing 401147, P. R. China

\section{References}

[1] P.J. Szakács G, Ludwig JA, Booth-Genthe C, Gottesman MM, Targeting multidrug resistance in cancer, Nat Rev Drug Discov 5(3) (2006) 219-34.

[2] P.M. Chaudhary, Expression and activity of P-glycoprotein, a multidrug efflux pump, in human hematopoietic stem cells, Cell 66(1) (1991) 85-94. 
[3] J.I. Fletcher, M. Haber, M.J. Henderson, M.D. Norris, ABC transporters in cancer: more than just drug efflux pumps, Nat Rev Cancer 10(2) (2010) 147-56.

[4] G.Z. Wang H, Liu X, Agarwal P, Zhao S, Conroy DW, Ji G, Yu J, Jaroniec CP, Liu Z, Lu X, Li X, He X, Targeted production of reactive oxygen species in mitochondria to overcome cancer drug resistance, Nat Commun 9(1) (2019) 562.

[5] I.L. S. Nobili, B. Giglioni, E. Mini, Pharmacological strategies for overcoming multidrug resistance, Curr Drug Targets 7(7) (2006)

[6] V. Juang, C.H. Chang, C.S. Wang, H.E. Wang, Y.A.-O. Lo, pH-Responsive PEG-Shedding and Targeting Peptide-Modified Nanoparticles for Dual-Delivery of Irinotecan and microRNA to Enhance Tumor-Specific Therapy, Small 15(49) (2019) e1903296.

[7] C.Y. Liu F, Li Y, Guo Y, Cao Y, Li P, Wang Z, Gong Y, Ran H, Folate-receptor-targeted laser-activable poly(lactide- co-glycolic acid) nanoparticles loaded with paclitaxel/indocyanine green for photoacoustic/ultrasound imaging and chemo/photothermal therap, Int J Nanomedicine 13(6) (2018) 5139-5158.

[8] M. Tan, W. Liu, F. Liu, W. Zhang, H. Gao, J. Cheng, Y. Chen, Z. Wang, Y. Cao, H. Ran, Silk Fibroin-Coated Nanoagents for Acidic Lysosome Targeting by a Functional Preservation Strategy in Cancer Chemotherapy, Theranostics 9(4) (2019) 961-973.

[9] S. Behzadi, W. Serpooshan V Fau - Tao, M.A. Tao W Fau - Hamaly, M.Y. Hamaly Ma Fau - Alkawareek, E.C. Alkawareek My Fau - Dreaden, D. Dreaden Ec Fau - Brown, A.M. Brown D Fau - Alkilany, O.C. Alkilany Am Fau - Farokhzad, M. Farokhzad Oc Fau - Mahmoudi, M. Mahmoudi, Cellular uptake of nanoparticles: journey inside the cell, Chem Soc Rev 46(14) (2017) 4218-4244.

[10] G. Alice, P. Capucine, C. Anne-Line, M. Claire, B. Anne-Sophie, P. Stéphane, V. Bernard, Biodegradable Polymeric Nanoparticles-Based Vaccine Adjuvants for Lymph Nodes Targeting, Vaccines 4(4) (2016) 34.

[11] T.F. Martens, K. Remaut, J. Demeester, S.C. De Smedt, K. Braeckmans, Intracellular delivery of nanomaterials: How to catch endosomal escape in the act, Nano Today 9(3) (2014) 344-364.

[12] L.I. Selby, C.M. Cortez-Jugo, G.K. Such, A.P.R. Johnston, Nanoescapology: progress toward understanding the endosomal escape of polymeric nanoparticles, Wiley Interdip Rev Nanomed Nanobiotechnol 9(5) (2017) 1452.

[13] T. Nicola, R. Roberta, N. Mariapaola, M. Barbara, F.A. Lisa, P.M. Adelaide, M.U. Maria, D. Cinzia, Role of Glutathione in Cancer Progression and Chemoresistance, Oxidative Medicine \& Cellular Longevity 2013 (2013) 972913.

[14] M.Q. Gong, C. Wu, X.Y. He, J.Y. Zong, J.L. Wu, R.X. Zhuo, S.X. Cheng, Tumor Targeting Synergistic Drug Delivery by Self-Assembled Hybrid Nanovesicles to Overcome Drug Resistance, Pharm Res 34(1) 
(2017) $148-160$.

[15] Y. Wang, W. Wu, J. Liu, P.N. Manghnani, B. Liu, Cancer-Cell-Activated Photodynamic Therapy Assisted by Cu(II) Based Metal-Organic Framework, ACS Nano 13(6) (2019) 6879-6890.

[16] S.C. Lu, Glutathione synthesis, Biochim Biophys Acta 1830(5) (2013) 3143-3153.

[17] Elie, Hatem, Nadine, El, Banna, Meng-Er, Huang, Multifaceted Roles of Glutathione and GlutathioneBased Systems in Carcinogenesis and Anticancer Drug Resistance, Antioxid Redox Signal 27(15) (2017) 1217-1234.

[18] Y.C. Ma, J.X. Wang, W. Tao, C. Sun, X.Z. Yang, Redox-Responsive Polyphosphoester-Based Micellar Nanomedicines for Overriding Chemoresistance in Breast Cancer Cells, ACS Appl Mater Interfaces 7(47) (2015) 26315-25.

[19] G.K. Balendiran, R. Dabur, D. Fraser, The role of glutathione in cancer, Cell Biochem Funct 22(6) (2004) 343-352.

[20] F.S. Yin X, Chi Y, Liu J, Sun K, Guo C, Wu, Estrogen-functionalized liposomes grafted with glutathioneresponsive sheddable chotooligosaccharides for the therapy of osteosarcoma, Drug Deliv 25(1) (2018) 900-908.

[21] Gergely, Szakács, Matthew, D., Hall, Michael, M., Gottesman, Ahcène, Boumendjel, Targeting the Achilles Heel of Multidrug-Resistant Cancer by Exploiting the Fitness Cost of Resistance, Chem Rev 114(11) (2014) 5753-74.

[22] G. Liang, Y. Zhu, D.J. Ali, T. Tian, H. Xu, K. Si, B. Sun, B. Chen, Z. Xiao, Engineered exosomes for targeted co-delivery of miR-21 inhibitor and chemotherapeutics to reverse drug resistance in colon cancer, J Nanobiotechnology 1(10) (2020) 18.

[23] R.A.-O. Bortolozzi, A. Luraghi, E. Mattiuzzo, A.A.-O. Sacchetti, A.A.-O. Silvani, G. Viola, Ecdysteroid Derivatives that Reverse P-Glycoprotein-Mediated Drug Resistance, J Nat Prod 83(8) (2020) 2434-2446.

[24] P. Huang, G. Wang, Y. Su, Y. Zhou, W. Huang, R. Zhang, D. Yan, Stimuli-responsive nanodrug selfassembled from amphiphilic drug-inhibitor conjugate for overcoming multidrug resistance in cancer treatment, Theranostics 9(20) (2019) 5755-5768.

[25] A. Bajaj, P. Kondaiah, S. Bhattacharya, Synthesis and Gene Transfection Efficacies of PEICholesterolBased Lipopolymers, Bioconjug Chem 19(8) (2008) 1640-1651.

[26] W.T. Godbey, K.K. Wu, A.G. Mikos, Poly(ethylenimine) and its role in gene delivery, Journal of Controlled Release 60(2-3) (1999) 149-160. 
[27] Ma, Enhancing endosomal escape for nanoparticle mediated siRNA delivery, Nanoscale 6(12) (2014) 6415-25.

[28] X. Xue, C. Qian, H. Fang, H. Liu, W. He, Photoactivated Lysosomal Escape of a Monofunctional PtII Complex Pt-BDPA for Nucleus Access, Angewandte Chemie International Edition 58(36) (2019) 1266112666.

[29] N. Kato, M. Nakamura, T. Uchiyama, $1 \mathrm{H}$ NMR studies of the reactions of copper(I) and copper(II) with D-penicillamine and glutathione, J Inorg Biochem 75(2) (1999) 117-121.

[30] H. Fan, G. Yan, Z. Zhao, X. Hu, W. Zhang, H. Liu, X. Fu, T. Fu, X.B. Zhang, W. Tan, A Smart Photosensitizer-Manganese Dioxide Nanosystem for Enhanced Photodynamic Therapy by Reducing Glutathione Levels in Cancer Cells, Angew Chem Int Ed Engl 55(18) (2016) 5477-82.

[31] C. Wang, F. Cao, Y. Ruan, X. Jia, W. Zhen, X. Jiang, Specific Generation of Singlet Oxygen through the Russell Mechanism in Hypoxic Tumors and GSH Depletion by Cu-TCPP Nanosheets for Cancer Therapy, Angew Chem Int Ed Engl 58(29) (2019) 9846-9850.

[32] K.H. Cowan, G. Batist, A. Tulpule, B.K. Sinha, C.E. Myers, Similar biochemical changes associated with multidrug resistance in human breast cancer cells and carcinogen-induced resistance to xenobiotics in rats, Proceedings of the National Academy of ences 83(24) (1986) 9328-9332.

[33]W.X. Li B, Chen L, Zhou Y, Dang W, Chang J, Wu C, Ultrathin Cu-TCPP MOF nanosheets: a new theragnostic nanoplatform with magnetic resonance/near-infrared thermal imaging for synergistic phototherapy of cancers, Theranostics 8(15) (2018) 4086-4096.

[34] L.L. Zhu,H.Y Zhou,H.Y. Xia, Z.G. Wang, H.T. Ran, P Li, J.L. Ren, Peptide-Functionalized Phasetransformation Nanoparticles for LIFU-Assisted Tumor Imaging and Therapy, Nano Lett 18(3) (2018) 1831-1841.

[35] H.Y Zhou, L.L. Zhu, Y. Tian, M. Wu, Y. Li, L.M. Deng, W. Jiang, W. Shen, Z.G. Wang, Z. Mei, P. Li, H.T. Ran, Z.Y. Zhou, J.L. Ren, Cell-penetrating Peptide-modified Targeted Drug-loaded Phase-transformation Lipid Nanoparticles Combined with Low-intensity Focused Ultrasound for Precision Theranostics against Hepatocellular Carcinoma, Theranostics 8(7) (2018) 1892-1910.

[36] X.C. Lu W, Zhang R, Shi L, Huang M, Zhang G, Song S, Huang Q, Liu GY, Li C, Receptor-mediated transcytosis: A mechanism for active extravascular transport of nanoparticles in solid tumor, Journal of Controlled Release 161(3) (2012) 959-66.

[37] L. Roth, V.R. Agemy L Fau - Kotamraju, G. Kotamraju Vr Fau - Braun, T. Braun G Fau - Teesalu, K.N. Teesalu T Fau - Sugahara, J. Sugahara Kn Fau - Hamzah, E. Hamzah J Fau - Ruoslahti, E. Ruoslahti, Transtumoral targeting enabled by a novel neuropilin-binding peptide, Oncogene 31(33) (2012) 3754-63. 
[38] L. Luo, C. Zhu, H. Yin, M. Jiang, J. Zhang, B. Qin, Z. Luo, X. Yuan, J. Yang, W. Li, Y. Du, Laser Immunotherapy in Combination with Perdurable PD-1 Blocking for the Treatment of Metastatic Tumors, Nat Commun 12(8) (2018) 7647-7662.

[39] W. Yue, L. Chen, L. Yu, B. Zhou, H. Yin, W. Ren, C. Liu, L. Guo, Y. Zhang, L. Sun, K. Zhang, Checkpoint blockade and nanosonosensitizer-augmented noninvasive sonodynamic therapy combination reduces tumour growth and metastases in mice, Nat Commun 10(1) (2019) 2025.

[40] S. Liu, S. Chen, W. Yuan, H. Wang, K. Chen, D. Li, D. Li, PD-1/PD-L1 interaction up-regulates MDR1/Pgp expression in breast cancer cells via PI3K/AKT and MAPK/ERK pathways, Oncotarget 8(59) (2017) 99901-99912.

[41] T.J. Curiel, Immunotherapy: a useful strategy to help combat multidrug resistance, Drug Resist Updat 15(1-2) (2012) 106-13.

[42] Y.X. Zhong, J. Xu, J. Zhou, J. Liu, M. Ye, L. Zhang, B. Qiao, Z.G. Wang, H.T. Ran, D.J. Guo, LowIntensity Focused Ultrasound-Responsive Phase-Transitional Nanoparticles for Thrombolysis without Vascular Damage: A Synergistic Nonpharmaceutical Strategy, ACS Nano 13(3) (2019) 3387-3403.

[43] C.G. Zhang , W.J. Zhu, B.G. You, Y. Liu, Z.Q. Yuan, W.L. Chen, J.Z. Li, X.F. Zhou, C. Liu, X.N. Zhang Distinctive polymer micelle designed for siRNA delivery and reversal of MDR1 gene-dependent multidrug resistance, Journal of Biomedical Materials Research Part B Applied Biomaterials 7(105) (2017) 20932106.

[44] Z.G. Yue, W. Wei, P.P. Lv, H. Yue, L.Y. Wang, Z.G. Su, G.H. Ma, Surface Charge Affects Cellular Uptake and Intracellular Trafficking of Chitosan-Based Nanoparticles, Biomacromolecules 12(7) (2011) 2440-6.

[45] L. Zhang, J. Sun, Y. Wang, J. Wang, X. Shi, G. Hu, Nonspecific Organelle-Targeting Strategy with CoreShell Nanoparticles of Varied Lipid Components/Ratios, Anal Chem 88(14) (2016) 7344-51.

[46] M. Mario, O. Idil, R. Cecilia, Z. Xingdong, L. Morten, H. Kerst-Jan, R.P. Coppes, E. Nikolai, M. Muriel, R. Fulvio, Chloroquine inhibits autophagic flux by decreasing autophagosome-lysosome fusion, Autophagy 14(8) (2018) 1435-1455.

[47] Q. Feng, X. Li, Q. Chen, J. Sun, X. Shi, B. Ding, H. Yu, Y. Li, X. Jiang, One-Step Microfluidic Synthesis of Nanocomplex with Tunable Rigidity and Acid-Switchable Surface Charge for Overcoming Drug Resistance, Small 13(9) (2017).

[48] Andre, E., Net, Lutz, Maedler, Darrell, Velegol, Tian, Xia, Eric, Understanding biophysicochemical interactions at the nano-bio interface, Nat Mater 8(7) (2009) 543-57.

[49] F. Hirschhaeuser, H. Menne, C. Dittfeld, J. West, W. Mueller-Klieser, L.A. Kunz-Schughart, Multicellular tumor spheroids: An underestimated tool is catching up again, J Biotechnol 148(1) (2010) 3-15. 
[50] G. Greczynski, L. Hultman, X-ray photoelectron spectroscopy: Towards reliable binding energy referencing, Progress in Materials ence 107 (2019) 100591.

[51] B. Ma, S. Wang, F. Liu, S. Zhang, J. Duan, Z. Li, Y. Kong, Y. Sang, H. Liu, W. Bu, Self-Assembled CopperAmino Acid Nanoparticles for in Situ Glutathione 'AND' H2O2 Sequentially Triggered Chemodynamic Therapy, J Am Chem Soc 141(2) (2019) 849-857.

[52] K.J. Koski, J.J. Cha, B.W. Reed, C.D. Wessells, D. Kong, Y. Cui, High-Density Chemical Intercalation of Zero-Valent Copper into Bi2Se3 Nanoribbons, J Am Chem Soc 134(18) (2012) 7584-7.

[53] X. Zhu, J. Li, P. Peng, N. Hosseini-Nassab, B.R. Smith, Quantitative Drug Release Monitoring in Tumors of Living Subjects by Magnetic Particle Imaging Nanocomposite, Nano Lett 19(10) (2019) 67256733.

[54] T.C. Chou, Drug combination studies and their synergy quantification using the Chou-Talalay method, Cancer Res 70(2) (2010) 440-6.

\section{Figures}



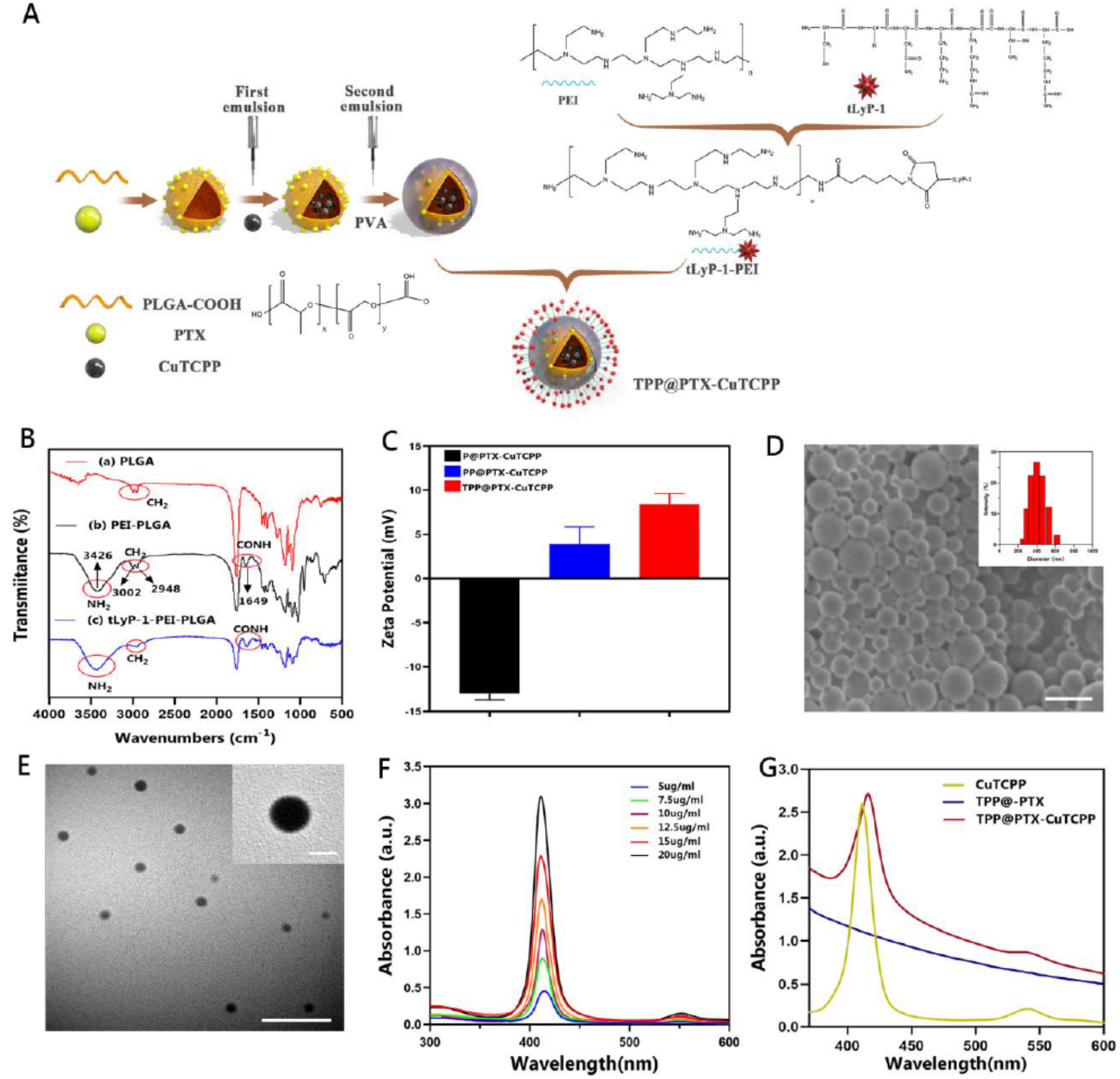

Figure 1

Synthesis scheme and characterizations of TPP@PTX-CuTCPP nanoparticle. (A) Schematic on the synthetic procedure of TPP@PTX-CuTCPP. (B) FTIR spectrum of different samples including PLGA (a) PEI-PLGA (b), and tLyP-1-PEI-PLGA (c). Based on curve $a$, curve $b$ and $c$ have characteristic peaks around 3426 and $1649 \mathrm{~cm}-1$ attributed to the stretching vibrations of the amide bond. (C) Zeta ptentials of P@PTX-CuTCPP, PP@PTX-CuTCPP, and TPP@PTX-CuTCPP. (D) An SEM image of TPP@PTX-CuTCPP, the scale bar is $0.5 \mu \mathrm{m}$ (inset: size distribution based on diameter, intensity (\%)). (E) A TEM image of TPP@PTX-CuTCPP NPs, the scale bar is $1 \mu \mathrm{m}$ (inset: scale bar is 200 nm). (F) UV-vis absorbance 
spectra of CuTCPP at elevated concentrations. (G) UV-vis absorbance of free CuTCPP, TPP@PTX, and TPP@PTX-CuTCPP.
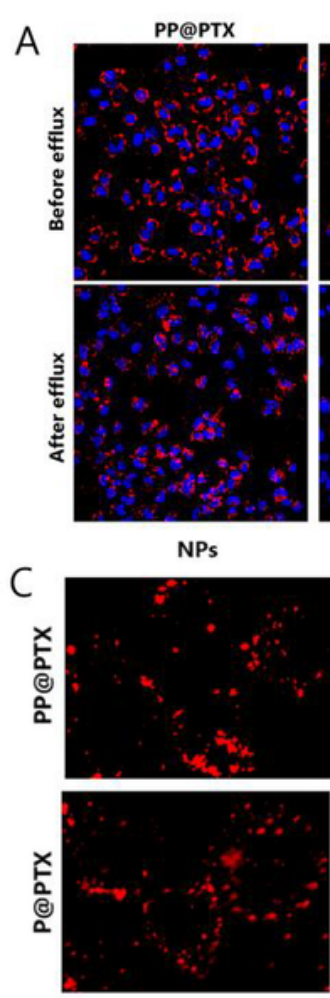

$\mathrm{D}$

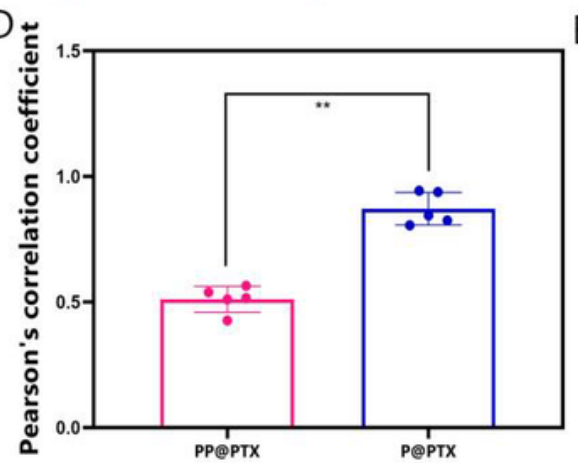

$\mathrm{F}$
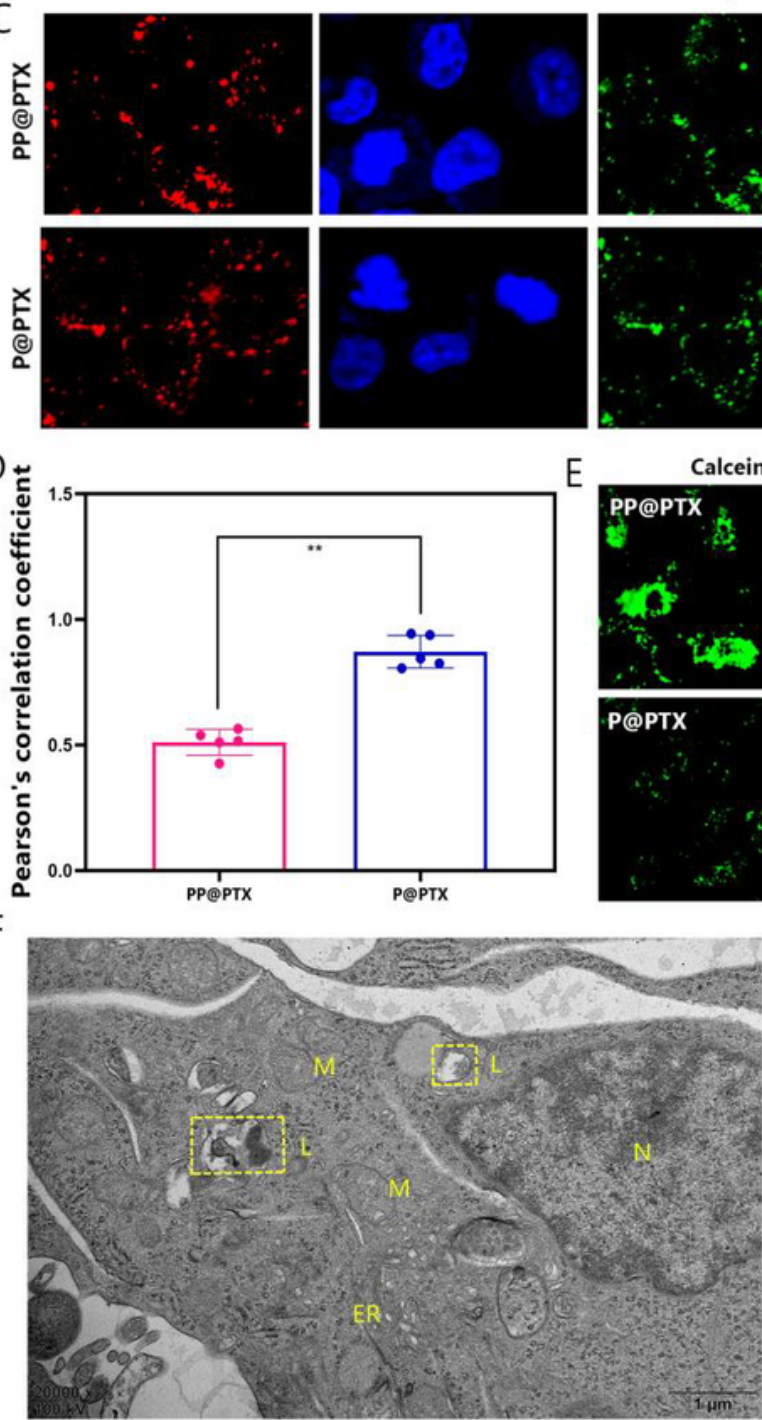

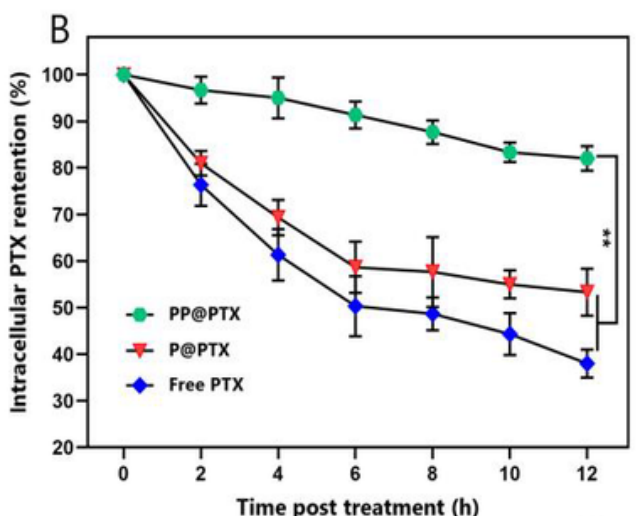

Endo/lysosome
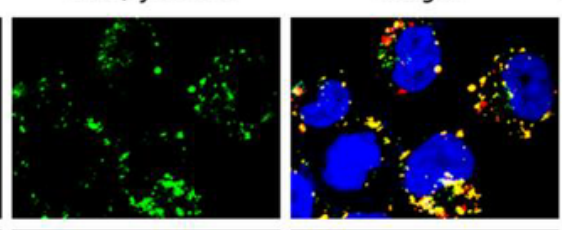

Co-location Scatterplots
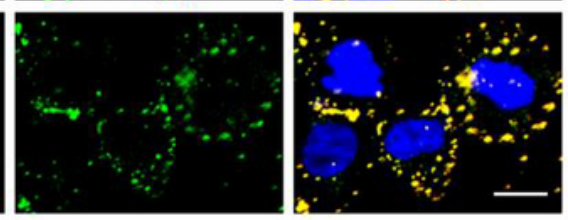

Bright Field
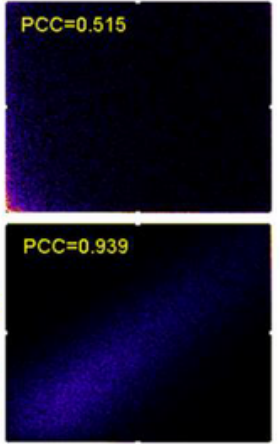

Merged
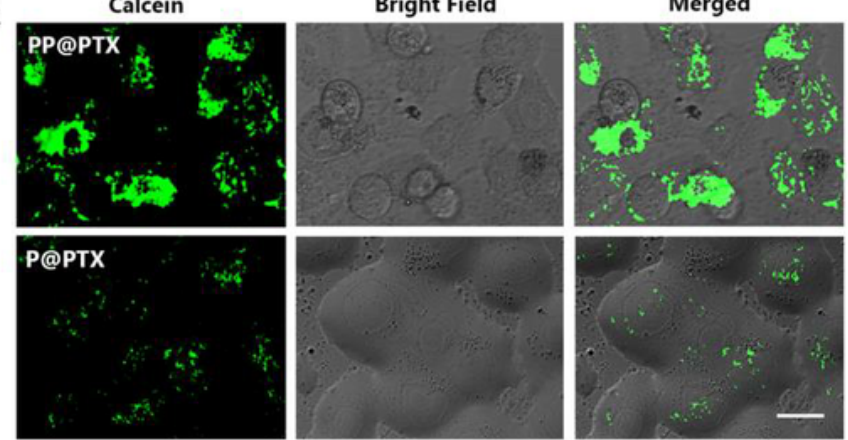

G

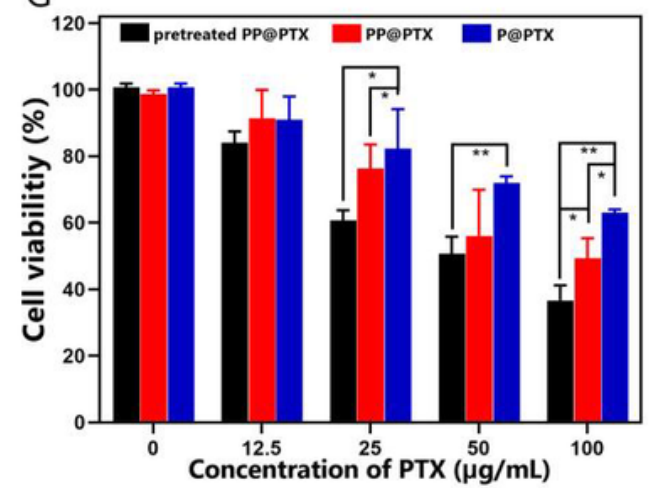

Figure 2

Intracellular accumulation and endo/lysosomal escape capability of the PEI-modified nanoparticle. (A) Intracellular PTX accumulation of MCF-7/Taxol cells coincubated with PP@PTX, P@PTX and free PTX before and after drug efflux. Blue fluorescence shows nuclei from DAPI; red fluorescence shows Dil- 
labeled PP@PTX NPs and P@PTX NPs; green fluorescence shows FTIC-labeled free PTX. The scale bar is $100 \mu \mathrm{m}$. (B) Intracellular PTX retention in MCF-7/Taxol cells at various times after Dil-labeled PP@PTX, P@PTX and free FITC-PTX treatment. (C) The colocalization of NPs and endo/lysosomes in MCF-7/Taxol cells after coincubation with Dil-labeld PP@PTX and P@PTX. Lysotracker stains the endo/lysosomes (green), and the NPs trapped in endo/lysosomes are labeled as yellow dots. Colocation scatterplots of PP@PTX and P@PTX vs endo/lysosomes are analyzed by Image J. The scale bar is $25 \mu \mathrm{m}$. (D) Corresponding Pearson's correlation coefficient (PCC) values of PP@PTX and P@PTX vs endo/lysosomes after $6 \mathrm{~h}$ coincubation, $\mathrm{n}=5$ per group. (E) integrity of endo/lysosomal membrane with calcein after conincubation with PP@PTX and P@PTX. The scar bar is $25 \mu \mathrm{m}$. (F) Bio-TEM images of MCF-7/Taxol cells incubated with PP@PTX for $6 \mathrm{~h}$. The ROI (yellow square in the image) reveals the membrane rupture and burst of lysosomes. L, lysosome; $\mathrm{N}$, nucleu; $\mathrm{M}$, mitochondria; $\mathrm{ER}$, endoplasmic reticulum. (G) Relative cell viability of MCF-7/Taxol cells after incubation with P@PTX, PP@PTX, and pretreated PP@PTX (pre-immersed in PBS at pH 6.3 for $2 \mathrm{~h}$ ) at different concentrations for $24 \mathrm{~h}$. All data are presented as mean $\pm S D,{ }^{*} p<0.05,{ }^{*} p<0.01$. 

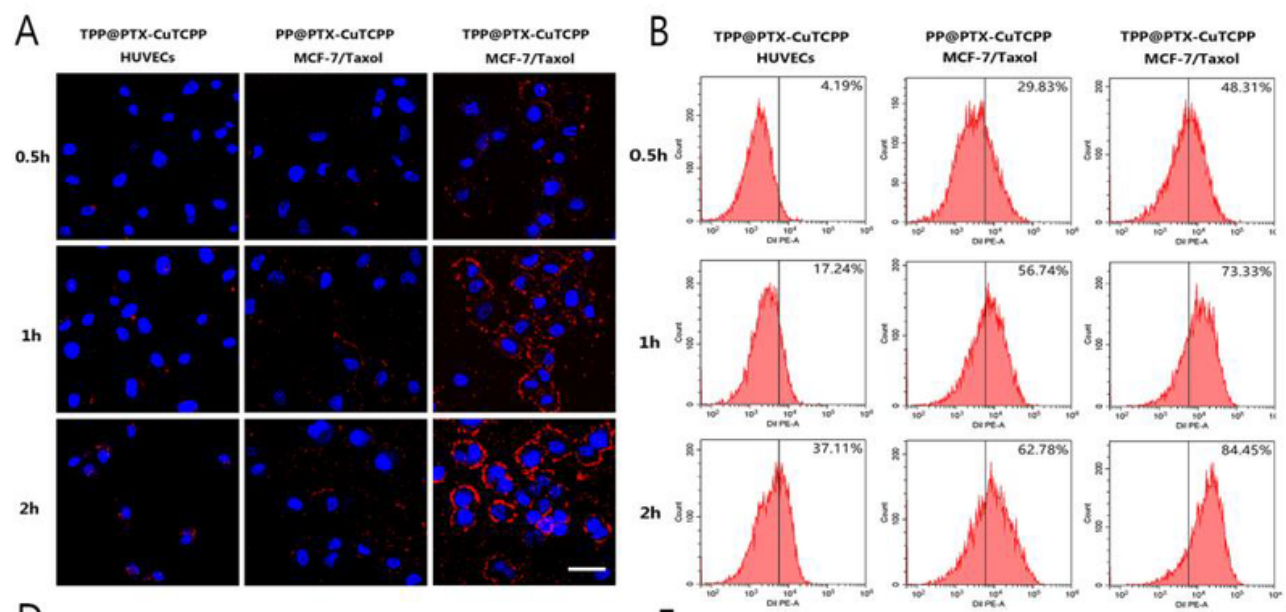

C
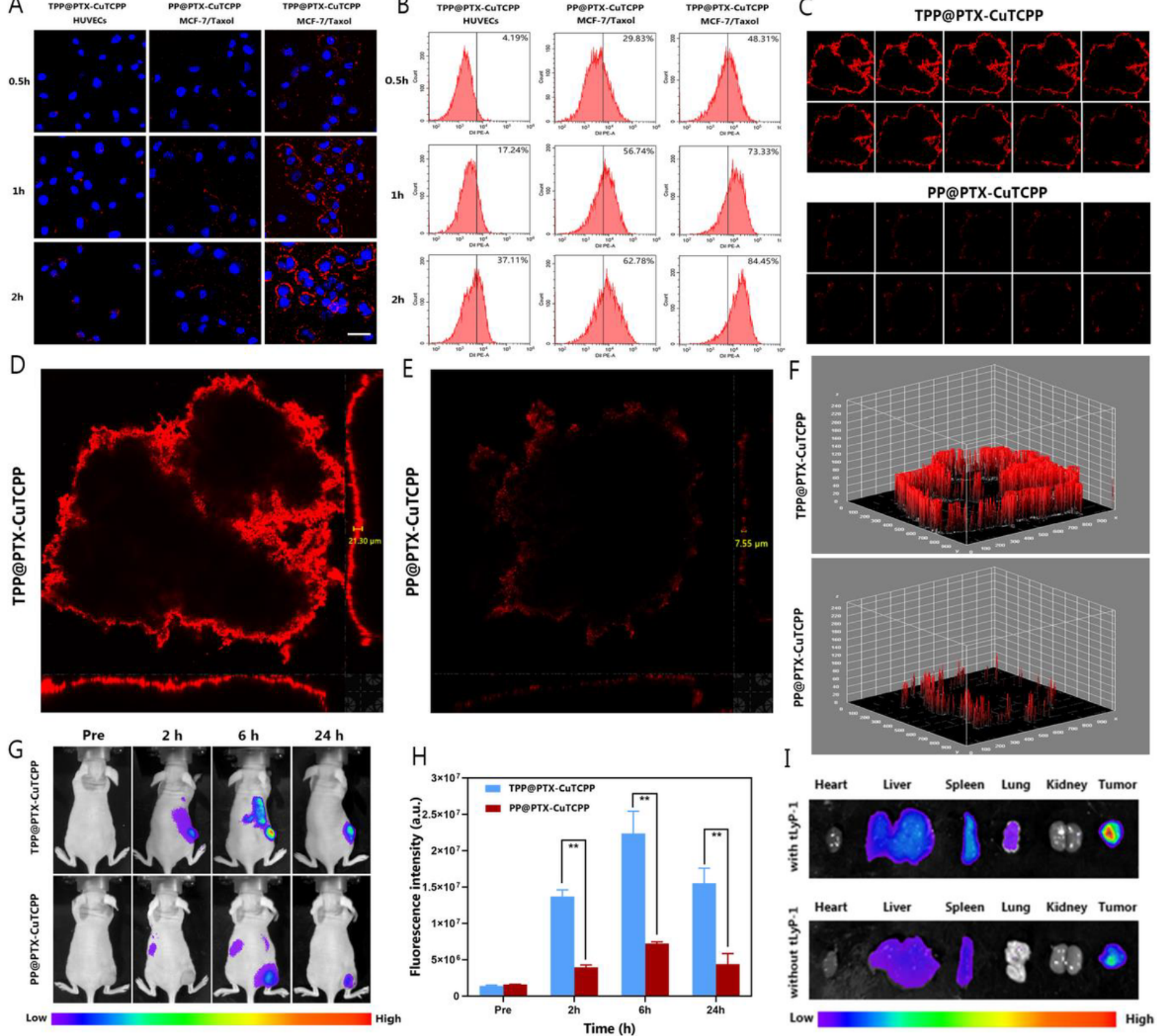

\section{Figure 3}

In vitro and in vivo targeting and penetrating behavior endowed by tLyP-1 peptide. (A) CLSM images of the MCF-7/Taxol and HUVECs cells coincubated with TPP@PTX-CuTCPP and PP@PTX-CuTCPP for $0.5 \mathrm{~h}$, $1 \mathrm{~h}$, and $2 \mathrm{~h}$, respectively. Red fluorescence shows Dillabeled PP@PTX and P@PTX NPs; blue fluorescence represents nuclei attributing to DAPI. The scale bar is $50 \mu \mathrm{m}$. (B) Flow cytometry analysis of MCF-7/Taxol and HUVECs cells treated with Dil-labeled TPP@PTX-CuTCPP and PP@PTX-CuTCPP for 0.5 h, $1 \mathrm{~h}$, and $2 \mathrm{~h}$, respectively. (C) Multiple level scan started at the bottom of the spheroid at $2 \mu \mathrm{m}$ intervals for the penetration of Dil-labeled TPP@PTX-CuTCPP NPs and PP@PTX-CuTCPP NPs. (D) and (E) Quantitative analysis of the penetration depth of TPP@PTX-CuTCPP (left) and PP@PTX-CuTCPP (right) in three-dimensional tumor spheroid models. (F) Surface plots based on three-dimensional reconstruction 
of the MCF-7/Taxol spheroid models incubated with TPP@PTX-CuTCPP NPs (upper) or PP@PTXCuTCPP NPs (lower) for 6 h. (G) In vivo NIR fluorescence images of tumors in MCF-7/Taxol tumor-bearing mice after intravenous injection of TPP@PTX-CuTCPP and PP@PTX-CuTCPP at different time points. $(\mathrm{H})$ Corresponding fluorescence intensity (tumor:background) of MCF-7/Taxol tumor-bearing mice administered two types of NPs. Data are presented as mean $\pm S D, n=3$ per group. (I) Ex vivo fluorescence images of major organs and tumors dissected from mice $24 \mathrm{~h}$ postinjection of NPs with and without tLyP-1.
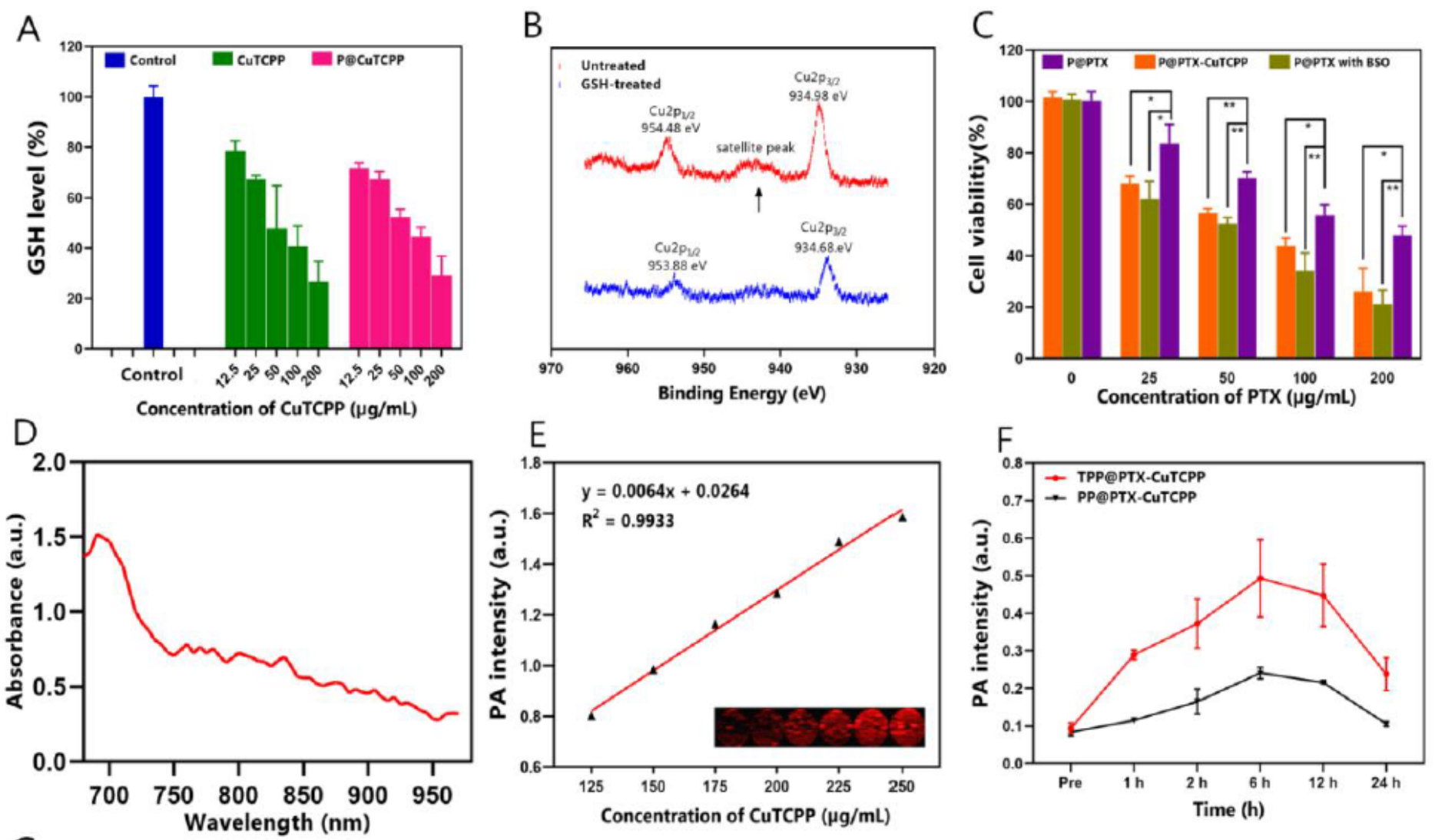

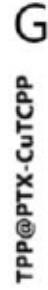
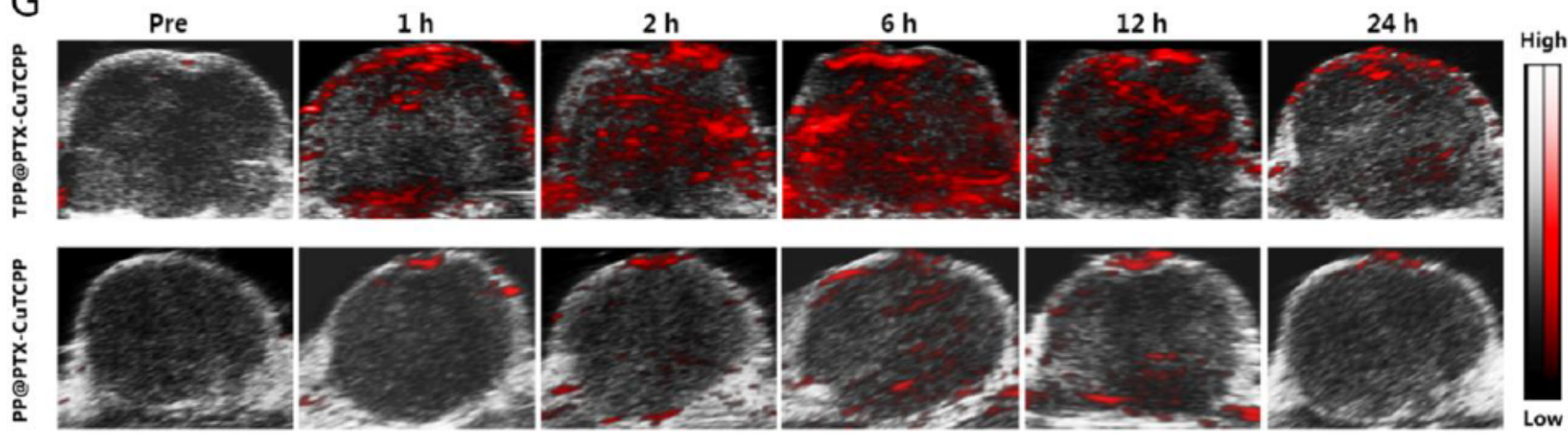

Figure 4

GSH depletion efficiency and photoacoustic contrast-enhanced imaging. (A) Residual GSH content in aliqutos determined by GSH assay kit after different corresponding concentration in P@CuTCPP and CUTCPP. (B) Detailed XPS spectrum of CuTCPP in the scanning regions of $\mathrm{Cu}$ before and after treating 
with GSH solution. The black arrow shows the position of the satellite peak. (C) Relative cell viability of MCF-7/Taxol cells after incubation with P@PTX, P@PTX-CuTCPP and P@PTX with BSO for $24 \mathrm{~h}$ at different concentrations. (D) The detection of optimal excitation wavelength by full spectrum scanning from $680 \mathrm{~nm}$ to $970 \mathrm{~nm}$ in PAl system. (E) In vitro PAl images and PAl values of TPP@PTX-CuTCPP at different CuTCPP concentrations. (F) Average photoacoustic intensity at tumor region after intravenous injection of NPs at different time intervals, $n=3$ per group. (G) In vivo PAl images of tumors in MCF7/Taxol tumor-bearing mice after intravenous injection TPP@PTX-CuTCPP and PP@PTX-CuTCPP at various time intervals. All data are presented as mean $\pm S D,{ }^{*} p<0.05,{ }^{*} \mathrm{p}<0.01$. 

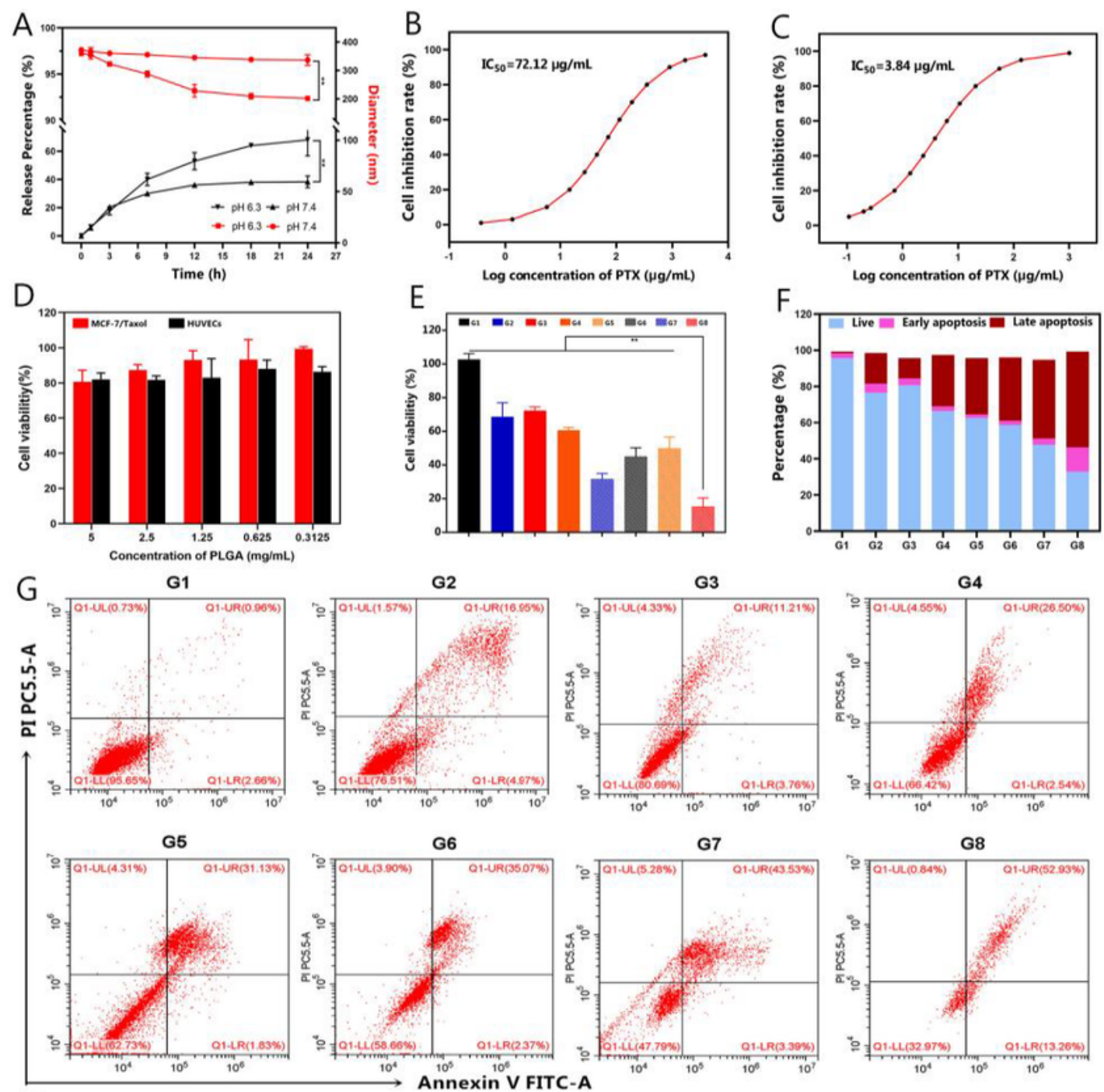

$\mathrm{H}$
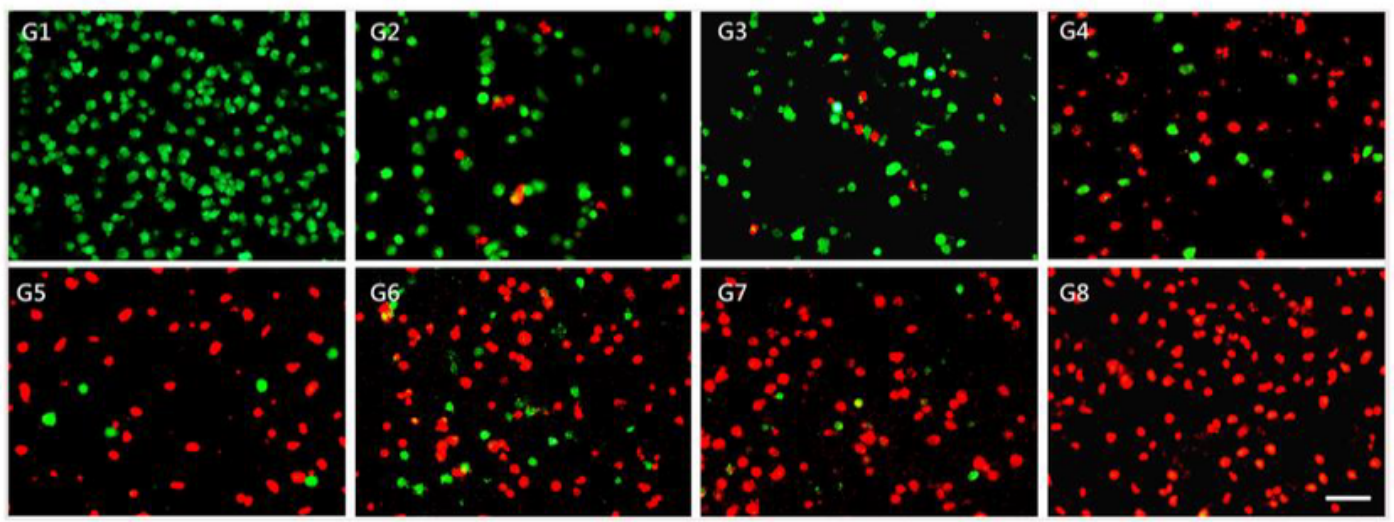

\section{Figure 5}

Synergistic chemotherapy efficacy of the various nanoformulations in vitro. (A) PH-responsive drug release changes and time-dependent changes of the size of TPP@PTX-CuTCPP under different pH values (6.3 and 7.4) at various time intervals. (B) and (C) IC50 curves of PTX against MCF-7/Taxol drug resistant cell line and MCF-7 parental cell line. (D) Biocompatibility of TPP@CuTCPP (PTX-free) against MCF7/Taxol and HUEVCs cells at varied concentrations for $24 \mathrm{~h}$ treatment. (E) Relative cell viability of MCF- 
7/Taxol cells after coincubation with corresponding formulations for $24 \mathrm{~h}$. The concentration of PTX is $50 \mu \mathrm{g} / \mathrm{mL}, \mathrm{n}=3$ per group. (F) and (G) Flow cytometric analysis on the apoptosis levels of MCF-7/Taxol cells of after corresponding treatments. $(\mathrm{H})$ CLSM images of MCF-7/Taxol cells stained by calcein AM and $\mathrm{PI}$ after different corresponding treatments. Green fluorescence represents live cell, red fluorescence represents dead cell. The scale bar is $100 \mu \mathrm{m}$. Note: G1-G8 represent control, free PTX, P@PTX, P@PTXCuTCPP, PP@PTX, TPP@PTX, PP@PTX-CuTCPP, and TPP@PTX-CuTCPP, respectively. All data are presented as mean $\pm S D$, ** $p<0.01$.
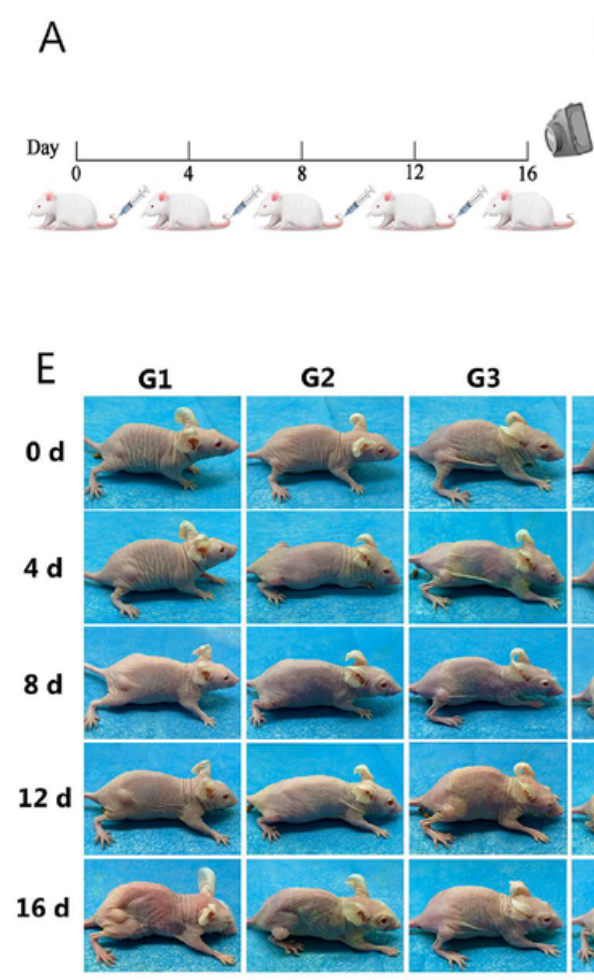

G2
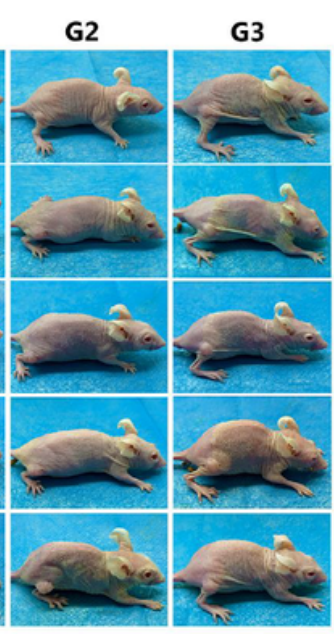

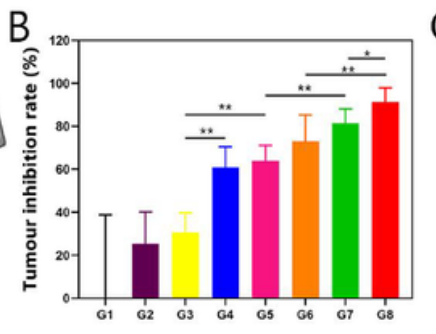

G4

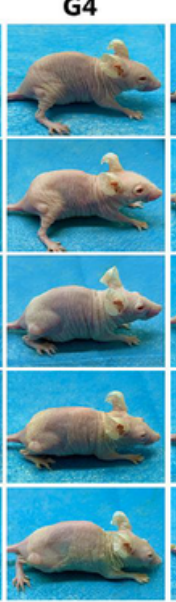

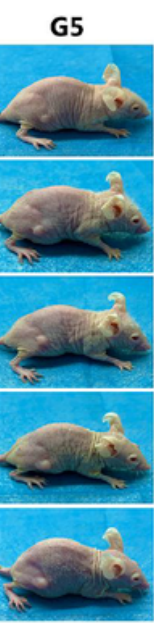

G6

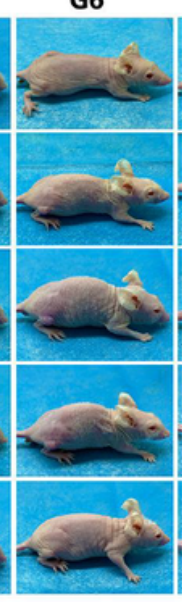

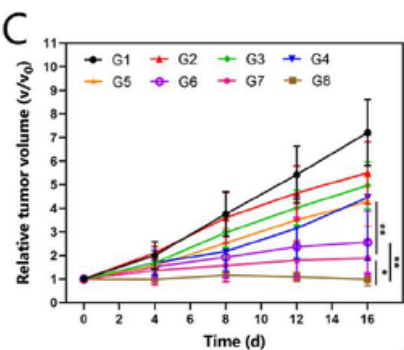

G8

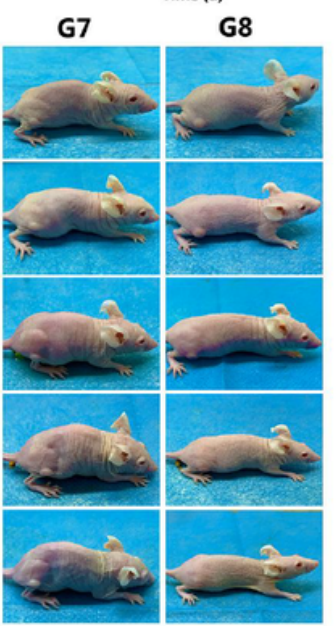

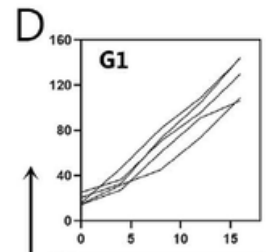
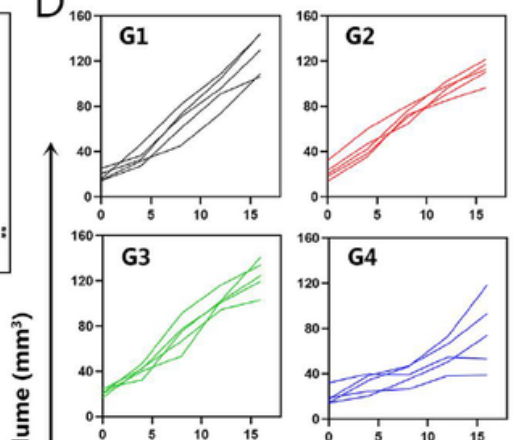
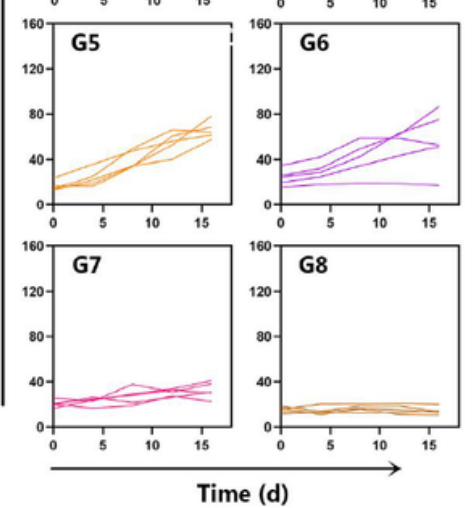

\section{Figure 6}

Evaluations on the combined effects for enhancing chemotherapy-based anti-tumor efficacy in vivo. (A) Schematic illustration of the treatment regimen. All groups were intravenously administered with different NPs on $0 \mathrm{~d}, 4 \mathrm{~d}, 8 \mathrm{~d}, 12 \mathrm{~d}$. (B) Tumor inhibition rates of eight groups after various treatments, $\mathrm{n}=5$ per group. (C) Relative tumor growth curves of different groups of MCF-7/Taxol tumor-bearing mice, $n=5$ per group. (D) Tumor growth curves of each mouse in G1-G8. (E) Representative digital pictures of MCF7/Taxol tumor-bearing mice of eight groups during $16 \mathrm{~d}$ period after intravenous administration of different formulations. Note: G1-G8 represent control, free PTX, P@PTX, P@PTX-CuTCPP, PP@PTX, TPP@PTX, PP@PTX-CuTCPP, and TPP@PTX-CuTCPP, respectively. All data are presented as mean \pm SD, ${ }^{*} p<0.05,{ }^{* *} p<0.01$. 


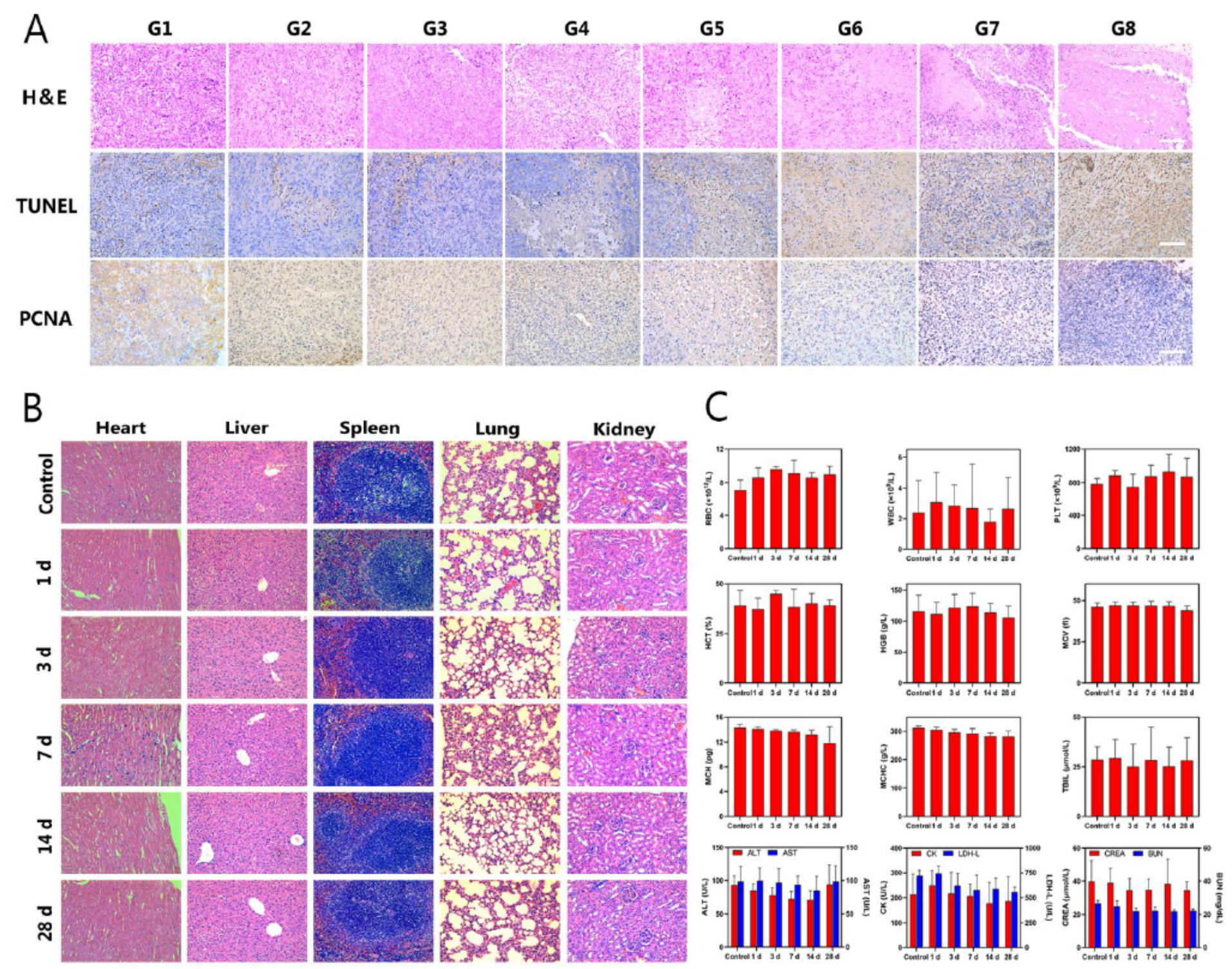

Figure 7

Pathological and blood biochemistry results of tumors and major organs in vivo. (A) H\&E, TUNEL, and PCNA staining on tumor sections from MCF-7/Taxol tumor-bearing mice after various treatments. The scale bar is $50 \mu \mathrm{m}$. (B) H\&E staining of the heart, liver, spleen, lungs, and kidneys in mice sacrificed at different time intervals (Control, 1 d, 3 d, 7d, 14 d, 28 d) after intravenous injection of TPP@PTX-CuTCPP NPs. The scale bar is $100 \mu \mathrm{m}$. (C) Blood biochemistry and complete blood analysis of Kunming mice sacrificed at different time intervals (Control, $1 d, 3 d, 7 d, 14 d, 28 d$ ) after intravenous injection of TPP@PTX-CuTCPP NPs. Data are shown as mean \pm SD, $n=4$ per group. Note: G1-G8 represent control, free PTX, P@PTX, P@PTX-CuTCPP, PP@PTX, TPP@PTX, PP@PTX-CuTCPP, and TPP@PTX-CuTCPP, respectively. All data are presented as mean \pm SD. 


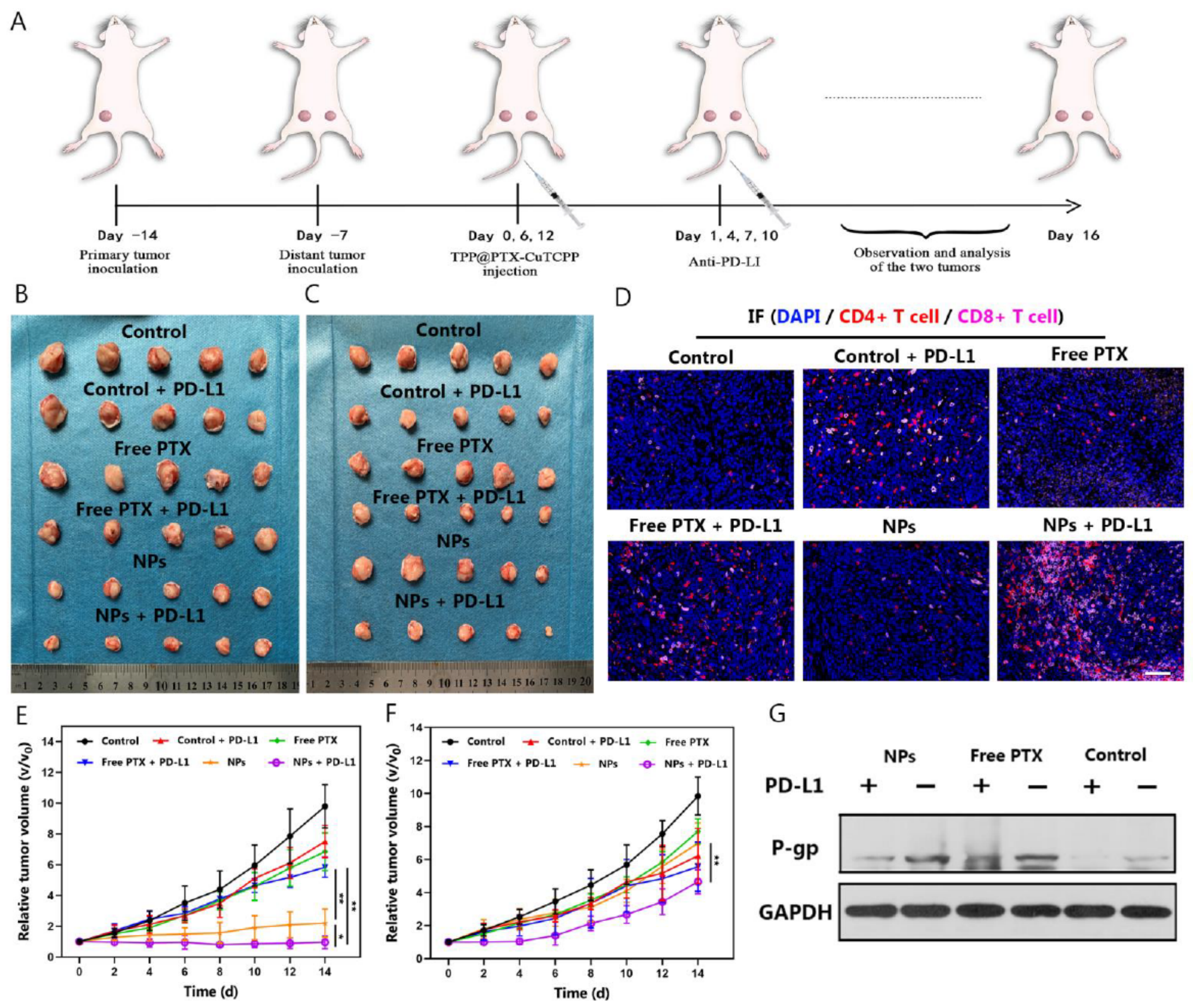

Figure 8

Anticancer efficacy and systemic immune responses initiated by immunochemotherapy in vivo. (A) Schematic illustration of the in vivo treatment design. (B) and (C) Representative digital pictures of 4T1 tumors of different groups at the end of $16 \mathrm{~d}$ post-treatment. (D) Immunofluorescence images of CD4+ (red) and CD8+ (pink) T cells in the distant tumors. (E) and (F) Relative tumor growth curves of primary and distant tumors after various treatments, $n=5$ per group. (G) Western blot for the detection of P-gp expression in groups with and without anti-PD-L1 treatment. The scale bar is $50 \mu \mathrm{m}$. All data are presented as mean $\pm S D, * p<0.05$, $* * p<0.01$.

\section{Supplementary Files}

This is a list of supplementary files associated with this preprint. Click to download. 
- GraphicalAbstract.tif

- Supportinformation.doc

- Scheme1.png 